\title{
The Cosmic Ray Energetics And Mass (CREAM) Instrument
}

\author{
H.S. Ahn ${ }^{\mathrm{a}}$, P. Allison ${ }^{\mathrm{b}}$, M.G. Bagliesi ${ }^{\mathrm{c}}$, J.J. Beatty ${ }^{\mathrm{b}}$, G. Bigongiaric ${ }^{\mathrm{c}}$, P. Boyle ${ }^{\mathrm{d}}$, J.T. Childers ${ }^{\mathrm{e}}$, N.B. Conklin ${ }^{\mathrm{f}}$, \\ S. Coutu ${ }^{\text {f }}$ M.A. DuVernois ${ }^{\mathrm{e}}$, O. Ganel ${ }^{\mathrm{a}^{*}}$, J.H. Han ${ }^{\mathrm{g} \dagger}$, J.A. Jeon ${ }^{\mathrm{g}}$, K.C. Kim ${ }^{\mathrm{a}}$, J.K. Lee ${ }^{\mathrm{g}}$, M.H. Lee ${ }^{\mathrm{a}}$, L. Lutz ${ }^{\mathrm{a}}$, \\ P. Maestro ${ }^{c}$, A. Malinin ${ }^{\mathrm{a}}$, P.S. Marrocchesi ${ }^{\mathrm{c}}$, S.A. Minnick ${ }^{\mathrm{h}}$, S.I. Mognet ${ }^{\mathrm{f}}$, S.W. Nam ${ }^{\mathrm{g}}$, S.L. Nutter ${ }^{\mathrm{i}}$, I.H. Park ${ }^{\mathrm{g}}$, \\ N.H. Park ${ }^{g}$, E.S. Seo ${ }^{\mathrm{a}, j}$, R. Sina ${ }^{\mathrm{a}}$, S.P. Swordy ${ }^{\mathrm{d}}$, S.P. Wakely ${ }^{\mathrm{d}}$, J. Wu$^{\mathrm{a}}$, J. Yang ${ }^{\mathrm{g}}$, Y.S. Yoon ${ }^{\mathrm{a}, \mathrm{j}}$, R. Zei ${ }^{\mathrm{c}}$ and S.Y. Zinn $^{\mathrm{a}}$ \\ ${ }^{a}$ Institute for Physical Science and Technology, University of Maryland, College Park, MD 20742, USA \\ ${ }^{b}$ Department of Physics, Ohio State University, Columbus, OH 43210, USA \\ ${ }^{c}$ Department of Physics, University of Siena and INFN, Siena 53100, Italy \\ ${ }^{d}$ Enrico Fermi Institute and Department of Physics, University of Chicago, Chicago, IL 60637, USA \\ ${ }^{e}$ School of Physics and Astronomy, University of Minnesota, MN 55414, USA \\ ${ }^{f}$ Department of Physics, Penn State University, University Park, PA 16802, USA \\ ${ }^{g}$ Department of Physics, Ewha Womans University, Seoul 120-750, S. Korea \\ ${ }^{h}$ Department of Physics, Kent State University Tuscarawas, New Philadelphia, OH 44663, USA \\ ${ }^{i}$ Department of Physics and Geology, Northern Kentucky University, Highland Heights, KY 41099, USA \\ ${ }^{j}$ Department of Physics, University of Maryland, College Park, MD 20742, USA
}

\begin{abstract}
The Cosmic Ray Energetics And Mass (CREAM) experiment is designed to investigate high energy $\left(10^{12} \sim 10^{15} \mathrm{eV}\right)$ cosmic rays over the elemental range from hydrogen to iron $(1 \leq Z \leq 26)$, through a series of long balloon flights. Originally planned to be flown on the first of the new Ultra Long Duration Balloon (ULDB) being developed by NASA, the CREAM instrument was launched as a long duration balloons (LDB) payload from McMurdo Station, Antarctica on December 16, 2004 and flew for a record-breaking 42 days. A second CREAM flight one year later lasted 28 days. The CREAM design is unique in that it obtains two independent energy measurements using a tungsten/scintillator sampling calorimeter and a Transition Radiation Detector (TRD) with up to four independent charge measurements of incident particles using a novel Timing-based scintillator Charge Detector (TCD), a plastic Cherenkov Detector (CD), scintillating fiber hodoscopes, and a Silicon Charge Detector (SCD). The energy limits are determined by trigger efficiency and telemetry bandwidth at the low end and by statistics at the high end.
\end{abstract}

\section{INTRODUCTION}

The origin, acceleration mechanism and propagation through the inter-stellar medium of cosmic-ray nuclei are some of the most fundamental problems in cosmology today. Theorists have developed a convincing model of acceleration by diffusive shock waves from supernova explosions that provides a natural explanation of the power law spectrum observed at energies above a few $10^{10} \mathrm{eV}$ and up to a few $10^{15} \mathrm{eV}$ [1]. This model predicts a sharp break at about $\mathrm{Z} \times 10^{14} \mathrm{eV}$, which has yet to be observed in any single-element spectrum. The all-particle spectrum observed by ground-based experiments shows a kink, the so-called 'knee', at about $3 \times 10^{15} \mathrm{eV}$, and some have reported an increase in the fraction of iron-like nuclei compared to proton-like nuclei around the knee [2,3], offering some support to the model. Direct measurement of a knee in the proton spectrum, however, would provide the most convincing evidence to support the model. To date, experiments such as ATIC [4] have made accurate direct measurements of the charge and energy of incident nuclei, but only up to about $10^{13} \mathrm{eV}$ due to the limited collecting power of these experiments. Others, such as JACEE [5] and RUNJOB [6] have made direct measurements to above $10^{14} \mathrm{eV}$, but with very limited energy and charge resolutions. TRACER [7] has flown a large TRD $\left(5 \mathrm{~m}^{2} \mathrm{sr}\right)$ for over 10 days, but with a charge range starting from oxygen, and with limited charge resolution preventing separation of rare nuclei. CREAM is poised to extend accurate direct measurements by more than an order of magnitude, to above the expected proton spectrum knee. Section 2 below lists the science and measurement objectives of CREAM. The instrument design and the reasoning that determined it are described in detail in section 3. Section 4 describes beam tests and calibration runs conducted from 2001 to 2003 at CERN, the European high energy physics lab, as well as the first flight. Conclusions are presented in section 5. 


\section{CREAM OBJECTIVES}

The science objectives of CREAM include the measurement of elemental spectra up to iron, determining if the proton spectral index is different than that of heavier nuclei, searching for potential spectral features such as a knee in the proton spectrum, and measuring the energy dependence of the flux ratio between secondary nuclei (e.g. boron) and primary nuclei (e.g. carbon). To achieve these objectives, the CREAM measurement objectives are as follows. An elemental coverage is required from hydrogen to iron inclusive ( $1 \leq \mathrm{Z} \leq 26)$, with a charge resolution sufficient to resolve each element in the range. The energy resolution is designed to be better than 50\% over the energy range, with an absolute calibration better than $10 \%$.

CREAM has a larger effective geometry factor for protons and helium than any previous, current, or planned instrument until ACCESS (Advanced Cosmic-ray Composition Experiment for the Space Station) or a similar detector is flown. Since no such instrument has been approved yet, none such is expected to be deployed until at least 2010. With its first flight CREAM has more than doubled the total world data of directly measured high energy $(>1 \mathrm{TeV})$ cosmic-ray nuclei with reasonable energy resolution, and has the first high statistics data sample allowing accurate boron to carbon ratio to be expanded to the $100 \mathrm{GeV} /$ nucleon region [8 and references therein]. The ultimate goal of the CREAM program is to collect at least 500 events each above $10^{14} \mathrm{eV}$, for protons, $\mathrm{He}, \mathrm{CNO}$, Ne-Si and the Fe group, and to produce elemental spectra for all nuclei up to iron, with the proton and helium spectra extending above $10^{15} \mathrm{eV}$.

\section{THE CREAM INSTRUMENT}

One of the greatest challenges in designing an instrument intended to directly measure high energy cosmic-ray particles is the enormous dynamic range needed to cover the energy range, along with the limited means of calibration. For low charge particles such as protons and helium nuclei, the only practical means of measuring energies above $10^{12} \mathrm{eV}$ is through the use of a calorimeter. This technique will work as well for heavier nuclei, but flight calorimeters are limited by the large mass required to allow a sufficient geometric acceptance for these lower flux particles. In addition, calorimeter calibration requires the use of accelerator beam particles of a known energy, which is limited by current technology to a few $10^{11} \mathrm{eV}$. This calibration was extended to about $10^{13} \mathrm{eV}$ by use of nuclear fragment beams (see Section 4 below). For nuclei with $\mathrm{Z}>3$, a TRD can measure the Lorenz factor $(\gamma)$, which when paired with knowledge of the particle mass, via charge identification, provides an energy measurement. The relatively low density of TRDs allows the larger geometric coverage needed for lower flux particles, with an acceptable detector weight. Since the TRD measures $\gamma$ rather than energy, one can calibrate this type of instrument using a variety of particles with varying masses (e.g. electrons, muons, pions and protons), so that a combination of beams can cover a range from $\gamma<10^{2}$ and up to $\gamma>10^{5}$, nicely covering the CREAM energy range of interest. However, TRDs cannot accurately measure high energy protons and helium nuclei, and their energy resolution is strongly energy dependent, making it more difficult to extract accurate spectra. By combining a calorimeter and a TRD in the same experiment, one can crosscalibrate between the two for a large sub-sample of events, for optimal energy determination [9]. Additionally, since the two detector types have different sources of systematic errors, combining the two reduces systematic uncertainties of the energy measurement.

One of the consequences of having a high density calorimeter as part of the experiment is the large number of secondary shower particles generated in the calorimeter absorber, some of which are scattered back up towards the top of the payload. This complicates the design of charge measurement devices that attempt to identify the incident primary particle. As the incident energy increases, so does the number of these back-scattered secondaries and their energy deposit in any charge detectors [10]. To overcome this 
challenge the CREAM design includes a large number of charge detectors, using a variety of measurement principles. These charge detectors are (from top to bottom) the TCD, the CD, the SCD, and the scintillating fiber hodoscopes (S0/S1 and S2). The TCD utilizes a fast readout system that takes advantage of the brief time interval between the primary's traverse and the arrival of the first secondaries to complete its charge measurement, thereby preventing the back-scatter noise from impacting its measurement of primary charge. The SCD is spatially divided into pixels of $2.12 \mathrm{~cm}^{2}$ area each, which minimizes the number of back-scattered particles hitting the same pixel that measures the primary particle. The $\mathrm{CD}$ only measures the Cherenkov radiation produced by relativistic particles, effectively rendering it blind to most back-scattered particles which have relatively low momenta. The fiber hodoscopes also use spatial segmentation, by reading out separate fibers with a cross section of only $2 \times 2$ $\mathrm{mm}^{2}$ and a length of $78 \mathrm{~cm}$ in S0/S1 $(64.6 \mathrm{~cm}$ in S2). Since back-scatter is concentrated near the primary path, most of the fiber area sees little if any back-scatter. For simulated protons in the TeV energy range the rates of charge mis-identification for protons using $3.0 \times 3.3 \mathrm{~cm}^{2}$ pixels have been estimated to be at the level of $\sim 1 \%$ [11]. For the $2.12 \mathrm{~cm}^{2}$ pixels of the CREAM SCD this rate should be even lower. The hodoscopes are used mostly for enhancing trajectory reconstruction to improve charge identification by other systems, which function is not affected by back-scatter to any great extent.

The CREAM instrument (Figs. 1,2) is comprised of several major detector sub-systems. At the top of the experiment is the TCD - a charge detector made up of two crossed layers of large-area thin scintillator paddles read out via fast, low-jitter photo multiplier tubes (PMT). Below the TCD are two TRD modules instrumented with thin-walled aluminized Mylar tubes containing a 95\% xenon, 5\% methane gas mixture. Between the TRD modules is a plastic Cherenkov detector to veto low-energy background particles present above Antarctica due to the low geomagnetic cutoff there. Below these is the calorimeter module, comprised of several parts. At the top of this module is the SCD, providing coverage for those particles that miss the TCD and enter the calorimeter module, as well as an independent charge measurement for those that traverse the TCD. Below the SCD is a four-layer scintillating fiber hodoscope (S0/S1), providing position measurements and a supplemental charge measurement, followed by a densified graphite target (T1), a two-layer fiber hodoscope (S2) for another position measurement and nuclear interaction detection, a second graphite target (T2), a single fiber layer shower detector (S3) read out by TCD-type PMTs to provide a reference time for the TCD, and finally a tungsten/scintillating fiber sampling calorimeter.

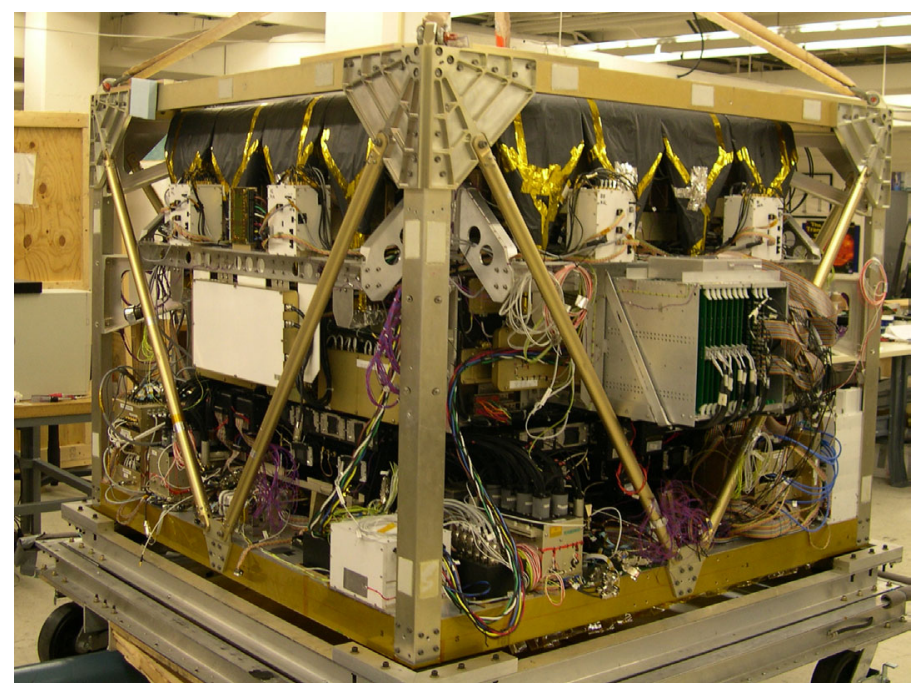

Figure 1. Photo of the CREAM Instrument during integration. 


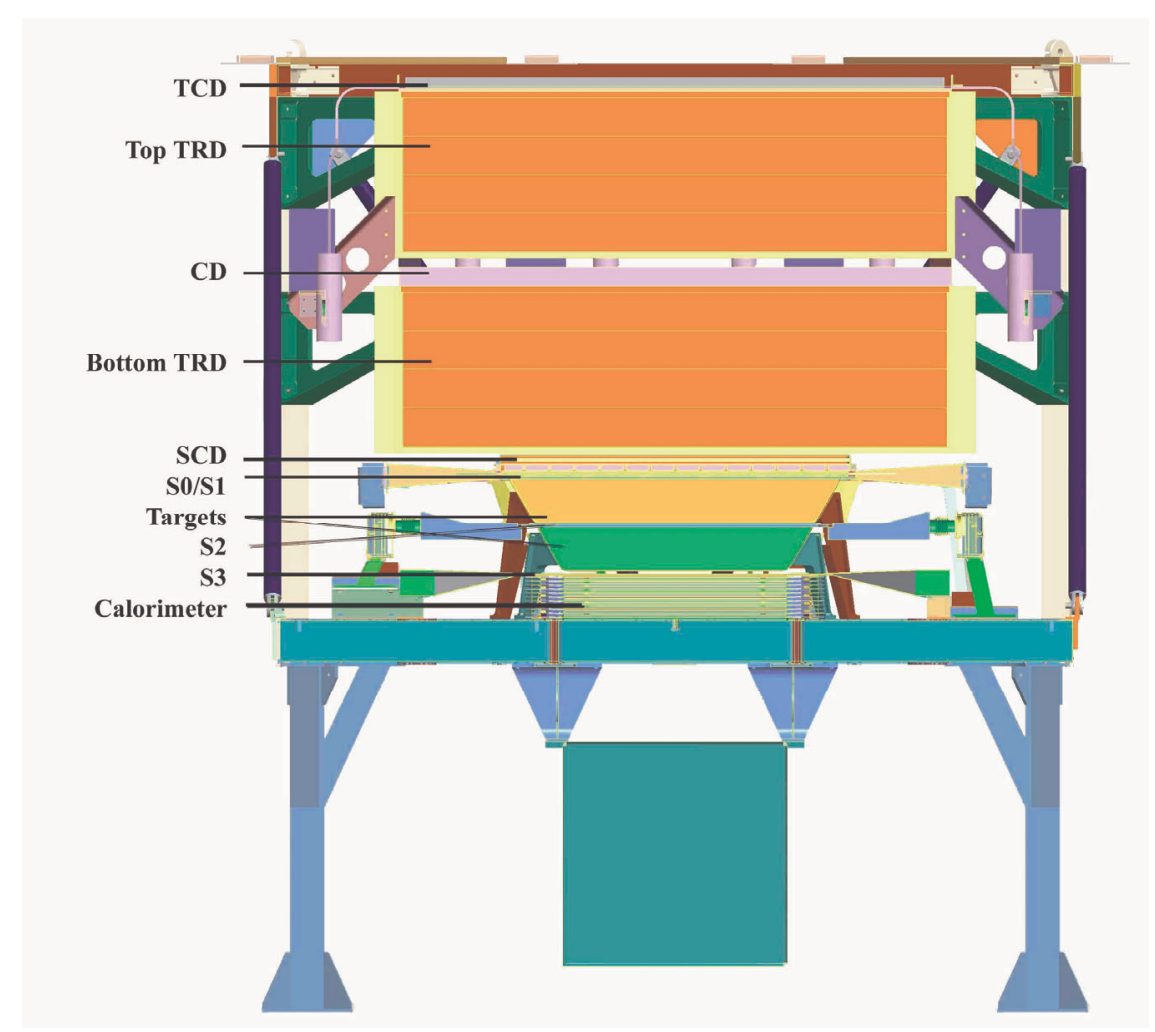

Figure 2. Schematic cross section drawing of major CREAM detector systems.

\section{$3.1 \mathrm{TCD} \& \mathrm{~S} 3$}

The most interesting events CREAM measures are those where a high energy nucleus enters through the top of the instrument, interacts in the graphite target and exits through the bottom of the calorimeter (section 3.5 below). In such events a large number of secondary shower particles are thrown back towards the upper detectors. This so-called back-scatter effect can interfere severely with accurate charge measurement of the primary nucleus, especially for lower-charged nuclei. Most charge detectors flown on similar experiments have thus been designed with fine spatial segmentation (e.g. pixels or strips) that minimize the area over which measurements are integrated (e.g. see sections 3.3 and 3.4, respectively, below). The TCD [12] takes advantage of an aspect of such events not previously utilized - their time structure. In CREAM, the arrival of the light signal from a primary nucleus at the TCD photo-multiplier tubes (PMTs) precedes the earliest possible arrival of a light signal from a back-scatter particle by $3 \mathrm{~ns}$. Thus, using an ultra-fast readout one can separate the primary signal from back-scatter noise without resorting to high channel counts.

The TCD is comprised of two layers, each with four $120 \times 30 \times 0.5 \mathrm{~cm}^{3}$ fast scintillator paddles (Saint Gobain BC-408, with vacuum compatible fluors) side by side. The paddles in the two layers are oriented with their long axes perpendicular to each other. Each paddle has an adiabatically shaped light guide (Fig. 3) glued to each of its two ends using Saint Gobain's BC-600 optical epoxy. The light guides gradually change cross sectional shape from a narrow rectangle at the scintillator glue-joint, to a nearly round cross section where they are glued to Photonis XP2020UR fast PMTs, without altering their cross sectional area. The light guides are UVA acrylic, where the UV absorption reduces the effects of Cherenkov radiation generated by particles traversing the acrylic, without significant reduction of the blue scintillation signal. The entire assembly is covered with crinkled aluminum foil and DuPont black Tedlar to assure light-tightness. Figure 4 shows a single (upside down) TCD paddle with light guides and PMT 
assemblies at its two ends. This arrangement produces at least 100 photo-electrons (p.e.) at each PMT from a singly-charged minimum ionizing particle (MIP) vertically incident at the center of a paddle. The charge resolution achievable with this technique is determined by fluctuations in ionization energy loss in the paddles, convolved with the scintillator response, and is $0.2 \mathrm{e}$ at oxygen, degrading to $0.35 \mathrm{e}$ at iron due to scintillator response saturation (Burke's law). The contribution from photon-statistics is negligible.

The high speed needed from the readout electronics requires borrowing from the techniques of time-offlight detectors. A series of 4 comparators with different thresholds are read out with fast (50 ps time resolution) custom fixed-threshold design time-to-digital converter (TDC) circuits. By reconstructing the time profile of signal increase in the first 3 ns one can measure the signal slew rate, and correcting for path length due to the angle of incidence, one can extract the square of the incident particle charge. For nuclei heavier than boron $(Z>5)$ the signal from the primary is expected to have a higher peak value than any back-scattered particle. Accordingly, an ultra-fast peak detector can capture this signal with a 3 ns rise time, which is then digitized using analog to digital converters (ADCs). The uncertainty in charge measurement contributed by this technique is still small with respect to that caused by the intrinsic fluctuations of energy loss in the scintillators. The TCD readout utilizes both systems to measure incident particle charge. The TCD data system consists of nine data acquisition units, or concentrators, running uClinux, each unit reading out two PMTs. Eight units read out the PMTs at the ends of the TCD scintillation counters, while the ninth unit reads out the two PMTs of the S3 detector. Each of the nine units houses two peak detector boards and two TDC boards, one each for each PMT. Each peak detector board contains four 12 bit AD7854L ADCs, to capture the peak voltage of four different dynode taps for increased dynamic range. The TDC boards also contain four 12 bit channels each, two to measure the rise time of the anode and two for the rise time of the $11^{\text {th }}$ dynode (of the 12 stage PMT, corresponding to about $1 / 5$ the anode gain). The thresholds on the anode were set to $100 \mathrm{mV}$ and $600 \mathrm{mV}$ throughout the flight, corresponding to $\sim 0.2$ MIPs and $\sim 1$ MIP, respectively. The $11^{\text {th }}$ dynode thresholds were changed during the flight to best tune for the boron and carbon peaks, and were ultimately left at $300 \mathrm{mV}$ and 1500 $\mathrm{mV}$, corresponding to about 3.5 MIPs and 20 MIPs, respectively.
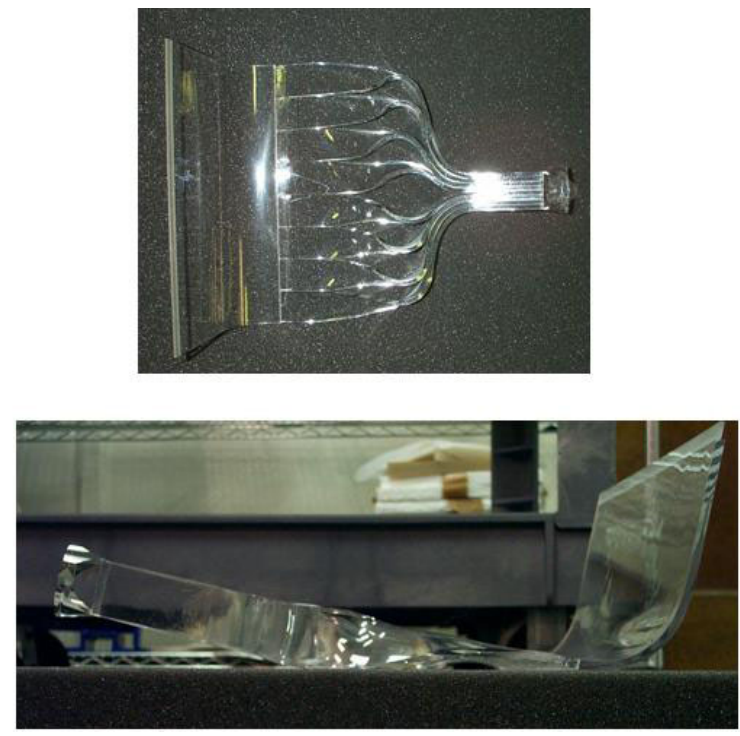

Figure 3. TCD adiabatic light guide.

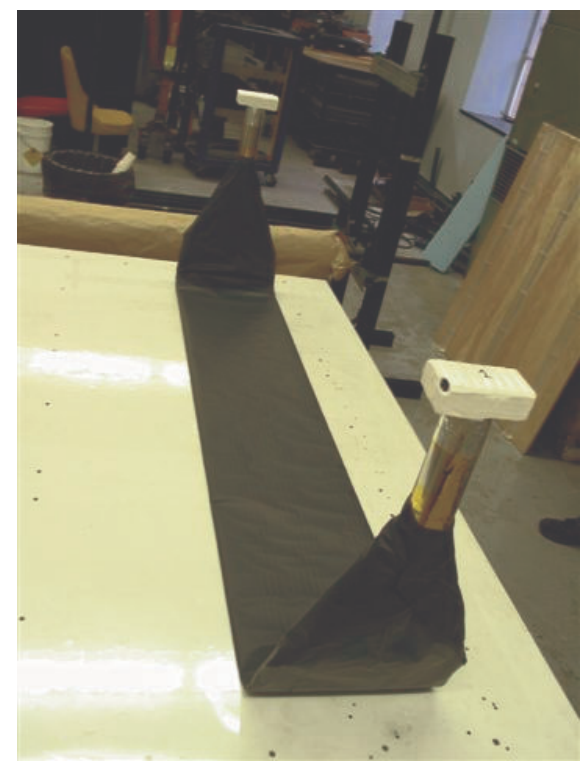

Figure 4. Individual TCD paddle with potted PMT assemblies at its ends.

To further reduce uncertainty due to back scatter, a layer of $2 \times 2 \mathrm{~mm}^{2}$ scintillating fibers (Saint Gobain BCF-12MC) is positioned directly over the calorimeter stack. This detector, labeled S3, measures the 
time at which back scattered particles start their way back to the TCD. From simple geometry and the speed of light one can then calculate the time at which the primary particle would have traversed the TCD scintillators, with an accuracy of $<0.1 \mathrm{~ns}$.

\subsection{TRD and Plastic Cherenkov Detector}

The electric field around a charged particle changes as it moves from one medium to another with a different dielectric constant. By forcing such a particle to experience many such transitions very rapidly one forces oscillations in the electric field strength, generating so called transition radiation (TR). One can select the types of materials and the typical thicknesses to tune the TR to the X-ray region. Detectors that use this technique and measure the X-rays are called transition radiation detectors (TRDs). TRDs measure the Lorentz factor $(\gamma)$ of incident particles, and can also reconstruct the particle trajectory through the detector.

The TRD (Fig. 5) [13] is comprised of two essentially identical modules, each with an active volume of $120 \times 120 \times 35 \mathrm{~cm}^{3}$ with a CD (see below) between the two. Each module has 4 layers of Dow Ethafoam 220 , serving the dual purpose of radiator and light-weight mechanical support. This polyethylene foam has closed cells with typical cell spacing of $870 \mu \mathrm{m}$, cell walls of $35 \mu \mathrm{m}$, and a density of about 0.036 $\mathrm{g} / \mathrm{cm}^{3}$. Each foam layer holds two layers of 32 thin-walled aluminized Mylar proportional tubes filled with a 95\% Xenon, 5\% Methane gas mixture, for a total of 512 tubes. Using such tubes obviates the need for a pressure vessel, significantly reducing the weight of the detector. The sense wire is maintained at $1.5 \mathrm{kV}$ relative to the grounded tube. The tube end pieces are designed especially to reduce the leak rate through the ends to less than that through the $75 \mu \mathrm{m}$ thick three layered Mylar walls using Resiweld 350 two-component epoxy.

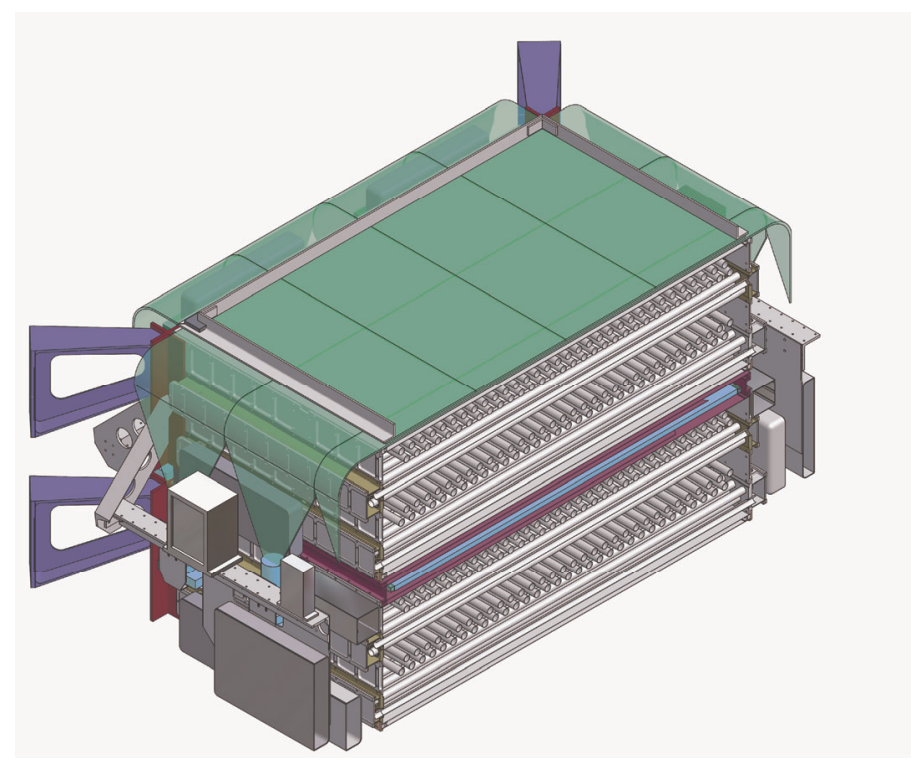

Fig. 5. TRD 3D schematic with cut through center plane to show tubes in both modules.

Measurements in the lab have shown that gas losses in a 100 day flight would be lower than the amount of reserve gas included in the system. In CREAM's first flight, over 42 days, no gas refill was needed. The manifolds were periodically connected to each other during the flight to balance pressure differences due to slightly different leak rates from different manifolds. However, the main valve to the reserve bottle was never opened (a schematic of the gas system is shown in Fig. 6). Pairing the tube layers allows full coverage in each orientation, as each pair is oriented perpendicular to the previous pair, providing 3- 
dimensional track reconstruction (Fig. 7). Charge pulses are generated when electrons are stripped, either by direct ionization of the gas by the primary incident nucleus or at higher energies by the TR X-rays. These stripped electrons are accelerated to the sense wire where they are measured by a readout system using Amplex 1.5 application specific integrated circuits (ASICs), with two ranges utilized to cover the 12-bit dynamic range needed to measure the energies from a lithium nucleus at $\gamma=10^{3}$ to a nickel nucleus at $\gamma=10^{5}$. The Amplex cards are mounted in boxes situated around the periphery of the TRD modules. The majority of the readout electronics are housed in a main electronics box. This box includes circuitry to control and read out the Amplex cards as well as circuitry to read out signals from the 8 CD PMTs. The box also provides a trigger input driven by the fast Cherenkov signal to form part of the TCD's ZHi trigger (see Section 3.8.3 below). The design uses a standard VME 6U form and backplane, but not VME electrical standards because of their large power draw which is not appropriate for balloon payloads. The bus interface is custom-designed for the TRD Amplex cards and the CD readout. The TRD is tuned for $10^{3}<\gamma<10^{5}$ and achieves an energy resolution of $15 \%$ for carbon nuclei and $7 \%$ for iron nuclei at $\gamma=$ 3000 [14]. An energy calibration of a single layer was carried out at CERN using beams of highly relativistic pions, protons, and electrons [14]. For particles with $Z>1$ one can scale by the square of the charge since the TR response depends only on linear electromagnetic properties of the materials involved.

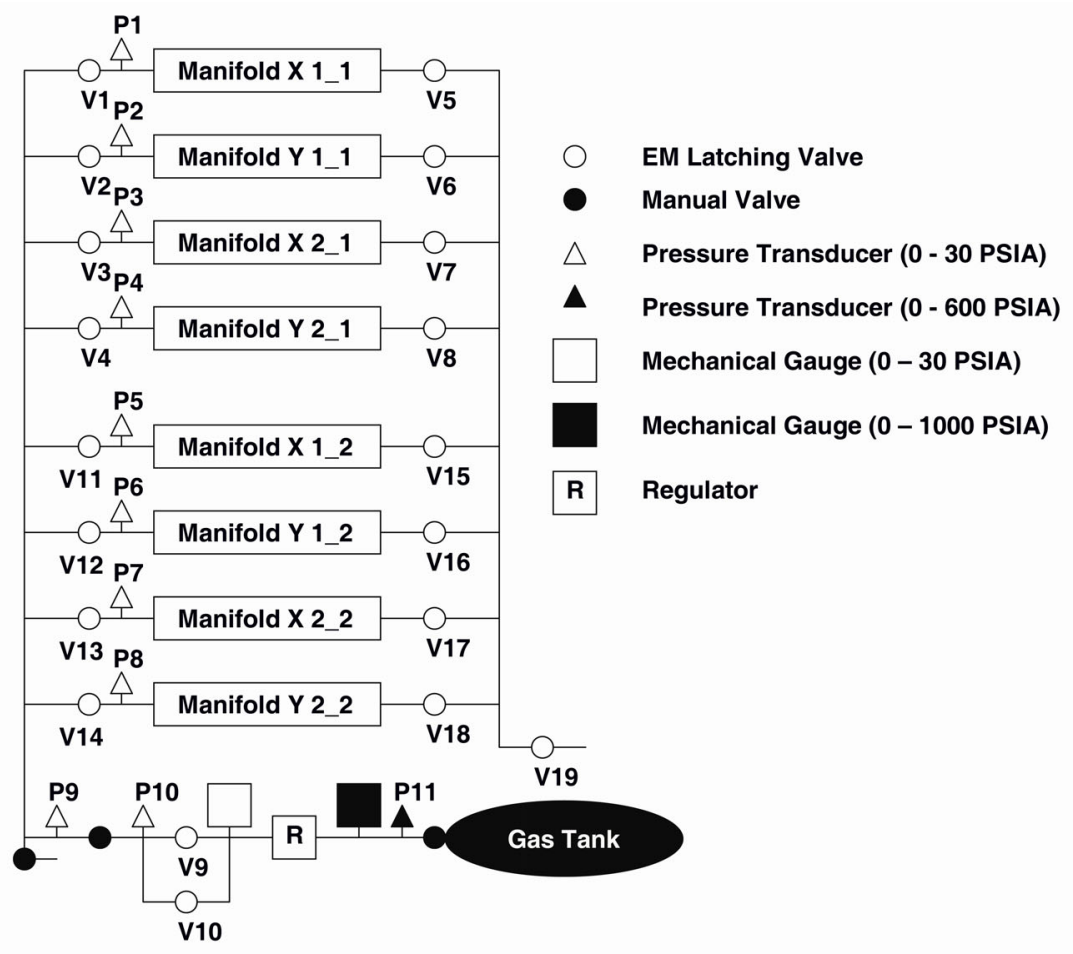

Figure 6. Schematic diagram of TRD gas system.

Between the two TRD modules, a Cherenkov layer is inserted to veto non-relativistic particles. This reduces the High- $Z$ trigger rate due to the low geomagnetic cutoff in Antarctica where CREAM flew, to an acceptable level of $<30 \mathrm{~Hz}$. This detector is read out by eight 0.5" Photonis XP1910 PMTs via four wave-length shifting bars, held against the radiator by a system of springs. The CD PMT signals are digitized using 12-bit peak sensing ADCs. Since the Cherenkov signal is also proportional to the square of the incident particle charge, this detector provides yet another independent charge measurement for relativistic charged particles. The readout is accomplished by custom-built shaping amplifiers connected to each PMT's tenth dynode. These are converted to digital counts by 12-bit ADCs in a custom circuit residing in the TRD main electronics box described above. The 8 PMT anode signals pass through a fast 
summing amplifier unit, the output of which is fed to a fast discriminator to provide an input to the TCD $\mathrm{Hi}-\mathrm{Z}$ trigger. When this trigger is not vetoed by the TCD, the TRD signals are read out.

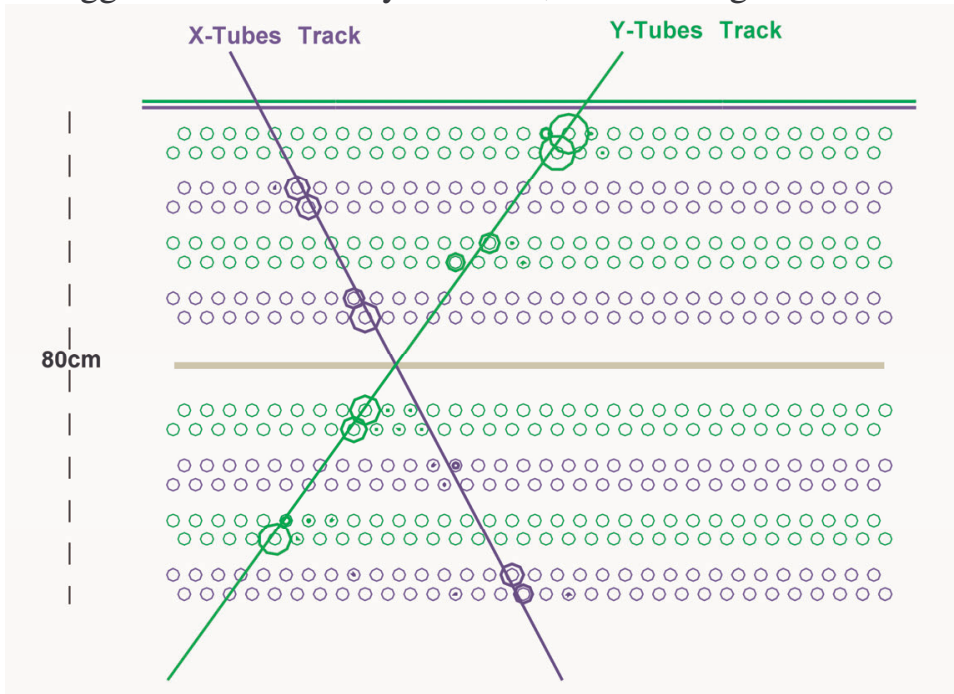

Figure 7. $\mathrm{X}-\mathrm{Z}$ and $\mathrm{Y}-\mathrm{Z}$ views of oxygen nucleus track through the TRD.

\subsection{SCD}

Positioned only $25 \mathrm{~cm}$ above the top of the calorimeter tungsten stack, the SCD uses fine segmentation to minimize back-scatter noise. The SCD [15] is comprised of 26 ladders arranged in an aluminum frame. Every ladder holds 7 sensors, each fabricated from a single high resistivity n-type 5" round silicon wafer, $380 \pm 15 \mu \mathrm{m}$ thick. Each sensor is segmented into a $4 \times 4$ array of $1.55 \times 1.37 \mathrm{~cm}^{2}$ pin-diode pixels, for a total of 2912 channels (Fig. 8). The sensors are slightly tilted and overlap each other in both lateral directions, providing full coverage in a single layer, with a $779 \times 795 \mathrm{~mm}^{2}$ coverage area. The analog readout electronics, incorporating a 16-channel Amplex CR-1.4A ASIC [16], are located directly under the sensors, with flexible Kapton cables connecting the two. The signals are digitized in 16-bit ADCs on readout boards on two opposite sides of the SCD (for 13 ladders each). To maximize geometric acceptance, the SCD thickness is kept at $7 \mathrm{~mm}$ for the detector, with an additional $15 \mathrm{~mm}$ height allowed for the $818 \times 818 \mathrm{~mm}^{2}$ mechanical enclosure.

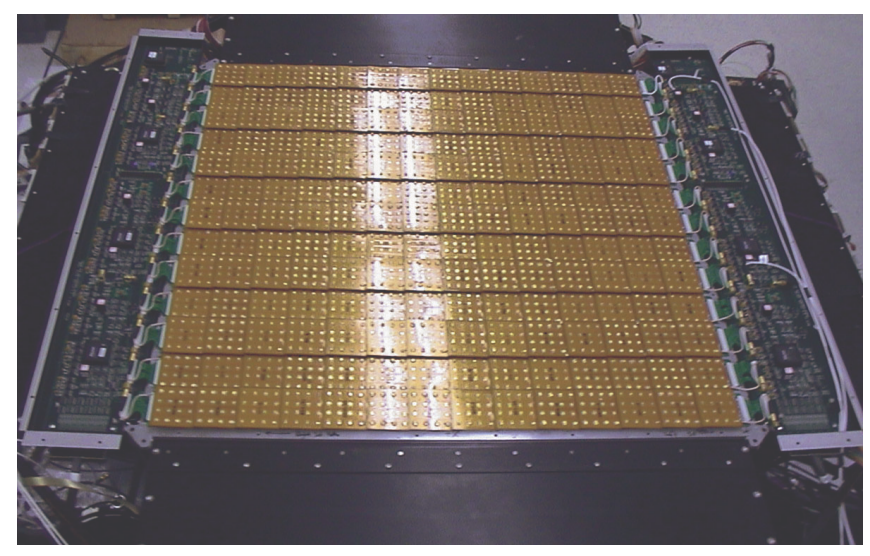

Figure 8. Photo of SCD with sensors and the two readout boards visible.

The SCD has a sufficient dynamic range to measure charges from $\mathrm{Z}=1$ (hydrogen) to 28 (nickel). With a pixel area of $\sim 2.12 \mathrm{~cm}^{2}$, the rate of charge mis-identification for low-Z nuclei at $10^{15} \mathrm{eV}$ is expected to be 
no more than $2-3 \%$. For higher $\mathrm{Z}$ nuclei, the impact of back-scatter noise on the $\mathrm{S} / \mathrm{N}$ ratio is lower, so mis-identification is less frequent. For lower energy showers fewer particles are back-scattered, so for that class of events too, the mis-identification fraction should be lower. A nearly fully populated SCD was placed in a beam of $158 \mathrm{GeV} /$ nucleon $\mathrm{A} / \mathrm{Z}=2$ nuclear fragments at CERN in November 2003, demonstrating that the design resolution was exceeded (see section 4 below). In flight, over 98\% of channels operated nominally, with the remainder masked as either dead or noisy [17].

\subsection{Fiber Hodoscopes}

The calorimeter (Section 3.5 below) is designed as thin as possible to increase the geometric acceptance. The trajectory reconstruction lever arm is lengthened by instrumenting the volume above and between the two graphite targets with fiber hodoscopes [18]. Directly above the upper target are 4 crossed active layers dubbed S0/S1, with each layer comprised of $3602 \times 2 \mathrm{~mm}^{2}$ scintillating fibers (Saint Gobain BCF$12 \mathrm{MC}$ ). Each fiber is polished on both ends and aluminized on one end to increase light signal reaching the readout end. This also increases the effective attenuation length and makes the fiber signal more uniform for particles traversing different positions along its length. Fibers are read out on alternate ends for mechanical convenience. The layer active area is approximately $78 \times 78 \mathrm{~cm}^{2}$, and the readout end of each fiber is glued to a short length of clear fiber of identical cross section (Saint Gobain BCF-98). The clear fibers are routed to square holes in 6 aluminum cookies per side (24 total), which are mounted on the end of a rectangular funnel-shaped light-tight enclosure (Fig. 9).

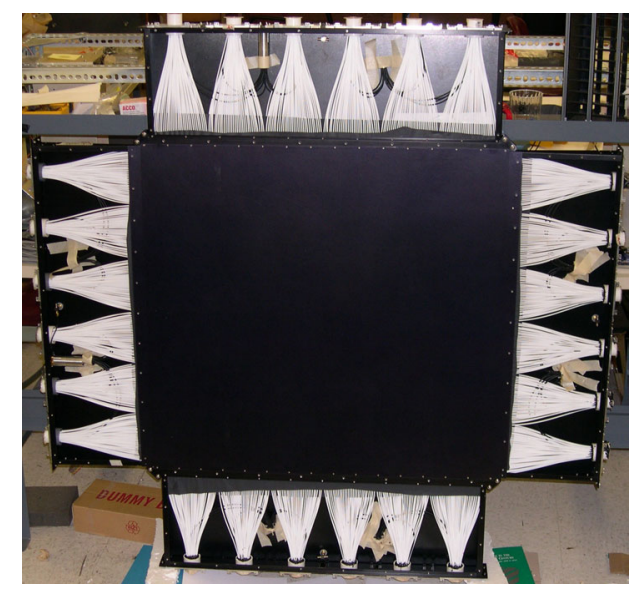

Figure 9. S0/S1 with top cover partially open to show clear fibers fanning in to HPD cookies.

Each cookie has a readout box with a 73-pixel hybrid photo-diode (Delft Electronics Products BV's PP0380BB HPD) mounted to it. Of the 73 pixels, 64 are read out [19] using four 16-channel Amplex CR-1.4A ASICs, with 30 pixels reading out fibers from the upper layer pair, 30 reading out fibers from the lower layer pair, and 4 reading out signals from blue light emitting diodes (LEDs). The LED signals are used to optimize pixel-to-fiber alignment, and in flight for aliveness testing and calibration. S0/S1 is used to aid track reconstruction and to provide supplemental charge identification. Another hodoscope with one crossed pair of fiber layers, S2 [20], is mounted between the upper and lower targets. The fibers in this detector are the same type and are read out in a similar manner, but with 3 HPDs per side (12 in total). In S2, only 3 pixels in each HPD are illuminated by LEDs. Since the targets are shaped as trapezoids which become narrower lower down, the active area of S2 is smaller, at $64.6 \times 64.6 \mathrm{~cm}^{2}$, with shorter scintillating fiber, at $64.6 \mathrm{~cm}$. S2 serves as another source of tracking information and helps determine if an interaction occurred in the upper target. Two neighboring S0/S1 HPDs and the S2 HPD below them are powered together by a single DEP PP0100BL $12 \mathrm{kV}$ high voltage power supply (HVPS). 


\subsection{Calorimeter and Graphite Targets}

With a calorimeter intended to measure the spectra of cosmic-ray nuclei, an energy resolution of 45-50\% is sufficient. The driving concern then becomes collection power, which is increased by maximizing the calorimeter area, keeping the thickness to the minimum required to contain the electromagnetic core generated at the first interaction, and preceding it with a flared low-Z target as a weight-efficient means to generate showers [10]. In CREAM, two $9.5 \mathrm{~cm}$ thick targets with a $30^{\circ}$ flare angle are mounted over the calorimeter (Fig. 10). These targets are comprised of blocks of densified graphite $\left(\rho=1.92 \mathrm{~g} / \mathrm{cm}^{3}\right)$ cemented in carbon/epoxy composite cradles. The targets induce nuclear interactions for approximately half of incident protons and up to $60 \%$ of incident Fe nuclei within the geometric acceptance, allowing the calorimeter to measure incident energy through the resulting showers. The calorimeter is a sampling tungsten/scintillating fiber device, with twenty (99.95\% pure) tungsten plates $50.1 \times 50.1 \times 0.35 \mathrm{~cm}^{3}$. Each tungsten plate is followed by a layer of $0.5 \mathrm{~mm}$ diameter scintillating fibers, arranged in 50 ribbons, each $10 \mathrm{~mm}$ wide, $503 \mathrm{~mm}$ long, and comprised of 19 fibers. This design allows a signal to be sampled approximately every radiation length $\left(\mathrm{X}_{0}\right)$, with a sampling fraction of $\sim 0.3 \%$. The small sampling fraction is sufficient for the multi-TeV showers being measured with the calorimeter, and helps minimize the stack height, maximizing geometric acceptance. A vertical depth of $20 \mathrm{X}_{0}$ (less than $10 \mathrm{~cm}$ !) provides sufficient containment of the electromagnetic core of the initial interaction, and contains the shower maximum for nuclei within the energy range of interest.

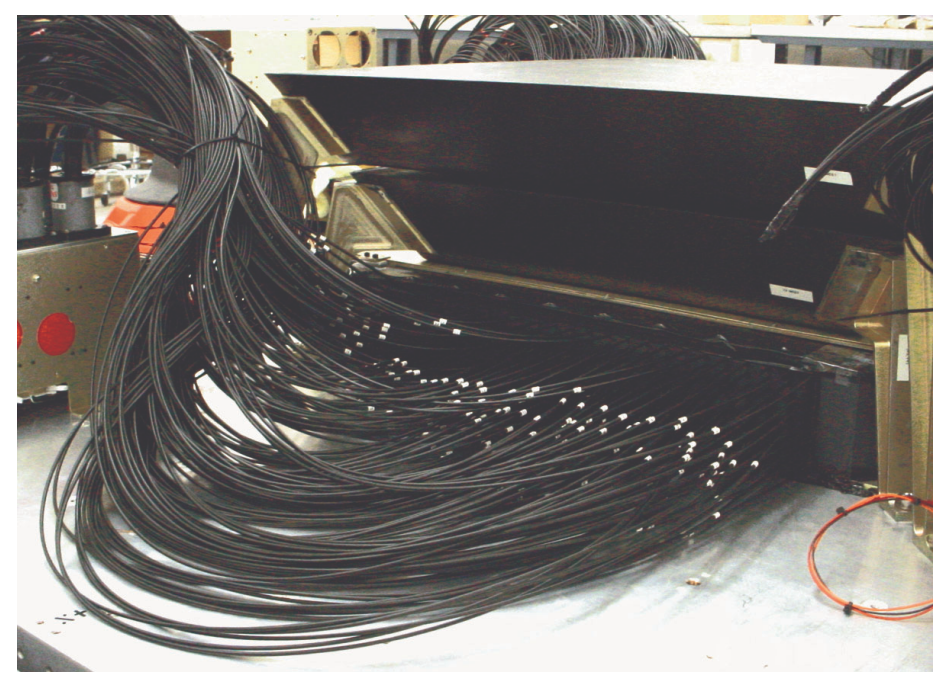

Figure 10. Calorimeter mounted under graphite targets during assembly of the calorimeter module. Shown in the foreground are the IFO fiber bundles carrying light signals through light-tight wall to a readout box.

The radiation length of tungsten, at $6.76 \mathrm{~g} / \mathrm{cm}^{2}$, is comparable to that of lead $\left(6.37 \mathrm{~g} / \mathrm{cm}^{2}\right)$, but with a density $70 \%$ higher, tungsten layers are $37 \%$ thinner per $\mathrm{X}_{0}$, again increasing the geometric acceptance. At the center of each but the top tungsten layer is a $0.9 \times 0.9 \times 500.9 \mathrm{~mm}^{3}$ stainless steel wire for mechanical support (needed to sustain the $10 \mathrm{~g}$ parachute opening shock at flight termination). At the corners of each tungsten plate are machined tabs with mounting holes used to bolt the $350 \mathrm{~kg}$ stack to an aluminum honeycomb pallet at the base of the instrument. The ribbons are polished at both ends, aluminized at the non-readout end, with neighboring ribbons read out in opposite directions, all for similar reasons as in the hodoscopes. The readout end of each fiber ribbon extends only $2 \mathrm{~mm}$ past the tungsten (to minimize the volume in which the calorimeter sampling fraction increases from $0.3 \%$ to near 100\%), and is glued into a slot in a UVT acrylic light guide. The light guide design changes its cross section from nearly rectangular at one end to nearly circular at its other end (Fig. 11a). A bundle of 48 thin (256 $\mu \mathrm{m}$ diameter) clear fibers, jacketed in black polyethylene (Industrial Fiber Optics LG48) is glued into the round end of the 
light guide, with the other end of the bundle separated into smaller bundles $(37+5+1)$ each read out separately. This optical division is used in addition to neutral density (ND) filters (Wratten 0.3 OD \& Wratten $0.8 \mathrm{OD}$, where OD is short for optical density) to provide low-range, mid-range, and high-range readouts. Half the ribbons in each layer are mounted on either side of the stainless steel wire, with each group wrapped in DuPont black Tedlar for light-tightness, and with ribbons in each layer oriented (with the steel wire) perpendicular to those of the nearest layers above and below them, providing 10 layers read out in one direction alternating with 10 in the other, allowing 3-dimensional track reconstruction. The ribbons of the lowest layer are mounted on an aluminum plate with identical shape and dimensions to those of the tungsten, allowing mechanical clearance for the light guides of the lowest active layer. For additional light-tightness a black Delrin plastic wall surrounds the calorimeter stack (Fig. 10), with machined grooves from above and below forming round holes for the jacketed fiber bundles, and with black GE RTV-103 silicone closing off any gaps in the wall around where the fiber bundles exit it. The thin clear fibers are routed into holes in 40 black Delrin cookies, with each cookie receiving the fibers from 25 ribbons of one readout direction of one layer (Fig. 11b). The low-range and mid-range fiber bundles are each routed into one hole per ribbon, and the high-end fiber bundles are collected in groups of five to reduce the channel count (the high-range readouts are expected to be used only for energy summation where segmentation is not important). Each cookie is potted with black epoxy (Appli-Tec 5105) for light tightness and to minimize optical cross-talk, and is mounted onto an aluminum alignment plate at the top of the readout box.

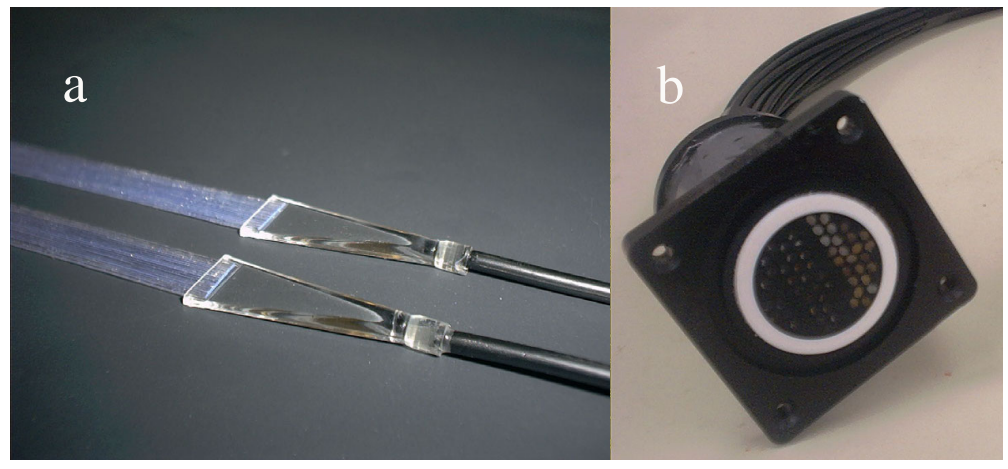

Figure 11. Calorimeter fiber ribbons with light mixers \& black jacketed clear fibers (a); and calorimeter cookie (b).

Each readout box (Fig. 12) mounts 10 HPDs powered by two HVPSs (all of the same types as in the hodoscopes), with readout circuitry [19] based on a pair of 32-channel IDEAS VA32HDR2/TA32C ASIC per HPD. The TA32C portion provides layer-based triggering (Section 3.8.2 below). Each HPD has 64 of its 73 pixels read out, covering the 25 low-range, 25 mid-range, and 5 high-range readouts of one side of one layer, 3 LED signals for alignment and in flight calibration, and 6 channels with no optical input to provide coherent electronic behavior information (e.g. pedestal drift, external RF noise, etc.). HPDs in the box on the opposite side of the instrument cover the alternate ribbons of the same layers. While the HPD is linear over a range of 1,000,000:1, more than sufficient for the 200,000:1 range needed for showers from $10^{12} \mathrm{eV}$ to $10^{15} \mathrm{eV}$, the VA32HDR2 ASICs linear dynamic range is just under 11 bits. Using three readout ranges (via the optical division described above), with a sensitivity ratio of $\sim 15: 1$ between low- and mid-range, and $\sim 36: 1$ between mid- and high-range, solves this problem, while providing sufficient overlap between the ranges for inter-range calibration using the data.

\subsection{High Voltage Systems}

At typical LDB and ULDB float altitudes the atmospheric pressure is in the $2-8$ mbar range, near the minimum of the Paschen voltage breakdown curve, where discharges can occur between nearby points with as little as $100 \mathrm{~V}$ potential difference. Some LDB experiments [4] use pressure vessels to avoid high 
voltage discharges. Designed for mission durations of over 100 days, it would have been difficult to maintain the pressure inside such a pressure vessel for CREAM. In addition, the more strict weight limits expected in ULDB missions guided the CREAM design to avoid the large weight penalty of such an approach by encapsulating high voltage systems instead. To protect against breakdowns, the HV portions of the HPDs and HVPSs circuits are potted using Conap EN-4/EN-11, with a semi-resistive coating to prevent surface charge accumulation.

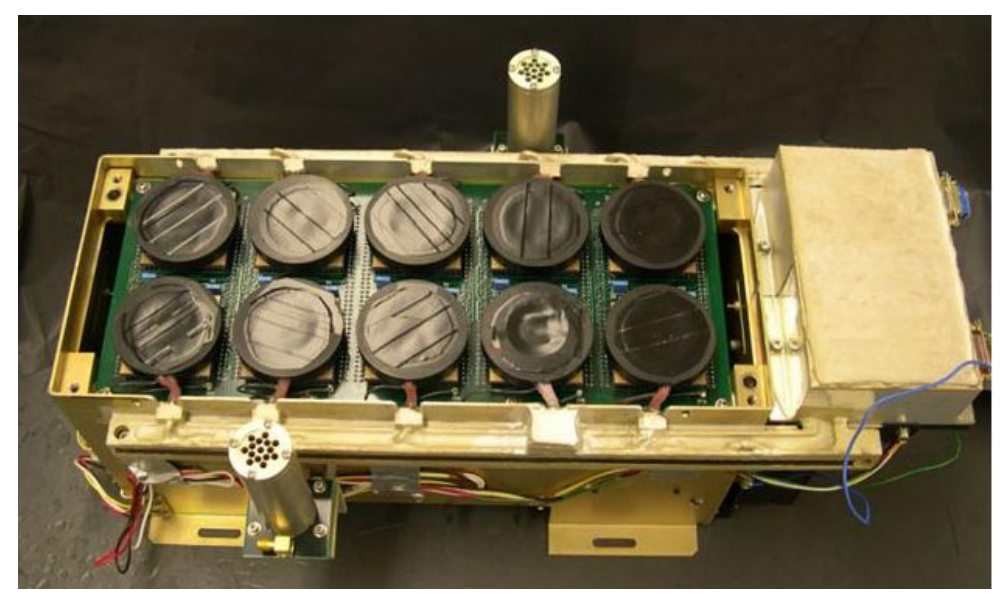

Figure 12. Calorimeter readout box with 10 HPDs (here covered for protection) \& EN-11 HV potting.

In the TCD, the four PMTs on each side of the instrument are supplied by a single Ultravolt potted power supply (model 4A12-P4-F). The same type power supply is used for the two S3 PMTs. The TCD PMTs are arranged such that all four on each side of the instrument are roughly gain-matched. Further gain matching is accomplished with series resistors. Each Ultravolt supply is potted in an aluminum box, with the board containing the matching series resistors potted in a separate box. The boxes and PMT voltage dividers are all primed with S11 primer and then potted with Emerson and Cuming Stycast 4640 White sealant with Catalyst 50. Extensive vacuum testing was performed for each circuit prior to flight, with no failures attributable to operation in the near-vacuum at float altitude. High-voltage vacuum-safe Reynolds coaxial cable (Type L) is used for all interconnections. The TRD and CD high voltage is provided by three Ultravolt 2a2a-p20 $(0-2 \mathrm{kV}, 20 \mathrm{~W})$ supplies, one for the top TRD module, one for the bottom, and one for the CD. Each is potted in an aluminum box with the same materials as the TCD HV supplies. The resulting units were tested under vacuum for over 30 days, assuring there would be no problems with these units during flight. The TRD high voltage was set to $1.4 \sim 1.5 \mathrm{kV}$ throughout the flight.

\subsection{Data Sparsification}

The CREAM instrument has 2560 calorimeter electronic channels, 2304 hodoscope electronic channels, 2912 SCD electronic channels, 144 TCD channels, 1024 TRD channels, and 8 CD channels, for a total of 8952 channels. With a trigger rate of $\sim 30 \mathrm{~Hz}$, sending 16 bits for each of these channels through the telemetry link for each event would require a bandwidth of nearly 4.3 Mbps. Using a high gain antenna in conjunction with the NASA Telemetry Data Relay Satellite System (TDRSS), CREAM has a bandwidth of $85 \mathrm{kbps}$ available, of which $35 \mathrm{kbps}$ is reserved for playback of archived data when the payload resumes telemetry after an outage, and $10 \%$ of the bandwidth is used for packet management. It is clear that the data rate must be reduced significantly to fit through the link. Since most particles traverse the TRD, TCD, CD, and SCD before interacting, and since the density of the tungsten keeps the lateral shower profile to within $\sim 5$ ribbons per layer for interacting particles, nearly all channels will only contain pedestal readings in most events. By using several algorithms (allowing thresholds to be set per channel as the channel's pedestal mean plus a constant, or its pedestal mean plus a multiple of its RMS 
width) in both hardware and software, the data stream is reduced to fit all of the $(\sim 0.3 \mathrm{~Hz})$ high energy shower events (see calorimeter trigger in Section 3.8.2), and up to $1 / 10$ of the $(\sim 30 \mathrm{~Hz})$ non-interacting high- $Z$ events (see high- $Z$ trigger in Section 3.8.3) in the available bandwidth. The remaining high- $Z$ events are written by the science flight computer (SFC, see Section 3.12) to a 43 GB flash drive, retrieved following the flight.

\subsubsection{Calorimeter Module Data Sparsification}

The three calorimeter module data sparsification boards, located near and powered by the SFC, perform two main functions for the nearly 7800 channels of the module - data collection and data sparsification. Event processing begins upon receipt of a signal from the trigger system or an external source. A programmable time delayed pulse is forwarded to the calorimeter and hodoscope HPD boxes, to activate the hold circuit within the ASICs of the readout systems. Additional delay time is added on the ASIC boards to match the specific peaking time of each ASIC. The data collection phase requires the generation of all timing signals needed to operate the analog output multiplexers within the ASICs, and the serial ADCs. The serial data from the ADCs is temporarily stored in serial-in/parallel-out shift registers, implemented in Actel field programmable gate arrays (FPGA). All ADCs controlled by a particular sparsification board are operated simultaneously. At the end of each conversion cycle, the sparsification process transfers all significant data to dual-port memories to be presented to the SFC. Each HPD pixel has a unique address as determined by the ASIC channel number and ADC number. The sparsification process sequences through each channel immediately after the A/D conversion, and compares the digital value stored in the shift register with a corresponding sparsification threshold for that channel using a simple magnitude comparator. If the data exceeds the threshold (by 5 counts for calorimeter, 10 for hodoscope, or 15 for SCD) the data and corresponding address are written to the dual port memory, and the memory address counter is incremented; otherwise nothing is written to memory and the next channel is evaluated. The sparsification threshold values are also stored in dual-port memory so they can be updated by the SFC as often as required. At the end of the cycle the total number of channels written to memory is stored in a 16 bit latch. This number, along with a unique 32 bit event number is written to the beginning of the memory buffer, and the buffers are swapped so the new one becomes available to the SFC. The data record consists of a number representing the total number of channels written to memory, a 32 bit event number, and a series of 32 bit data points, each consisting of 16 bits of ADC data and a 16 bit address. The sparsification boards provide trigger-disable outputs to the Master Trigger board to indicate a conversion is in process and triggers are not accepted. An I/O port is used to indicate to the computer when new data is available, and for the computer to acknowledge when it is finished reading the data. Sparsification thresholds are automatically updated (within commandable limits) by the computer. The threshold of each channel is determined by one of two algorithms, set by command to the one judged to best fit its behavior. The first algorithm checks the pedestal mean and RMS width of each channel periodically (every 5 minutes in flight), and sets the threshold to a commandable multiple of the pedestal RMS width above the mean pedestal. The alternate algorithm sets the threshold to a commandable number of ADC units above the mean pedestal value. These values are stored in a dual port memory chip physically located on a PC-104 interface board (Section 3.12 below). The memory sections are physically split between the two boards in this manner such that only data is presented across the interface, without any fast signals or edge dependent signals at the interface. The sparsification boards accept commands from the command processor, including hold circuit delay time, sparsification on/off, calibration mode on/off, and ASIC multiplex channel selection while in calibration mode.

\subsubsection{TCD Data Sparsification}

Each electronics unit reads out two neighboring PMTs using 16 channels (8 ADC and 8 TDC). Including the electronics for the S3 detector, 72 ADC channels and 72 TDC channels are read for each event, for a 
total of 144 channels. TDC channels without a valid bit (generated when the threshold for that channel is crossed) are not stored. Also, TDC and ADC channels with values below a set cut off are not stored, with the cut off set just above pedestals. The remaining channels are packed into a variable-length data structure and stored.

\subsubsection{TRD Data Sparsification}

There are 512 tubes in the TRD, each with two 8-bit numbers recording the signal amplitude in a highgain and a $(\times 12$ lower) low-gain channel. Only signals with sufficiently large amplitudes are recorded onto the onboard flash drive. A subset ( 10\%) of the TRD data is sent through the telemetry downlink to verify data quality during the flight. The signals to be recorded are identified by a hardware system which only passes through signals greater than the pedestal mean value plus a fixed number of ADC counts (set at 3 for flight). The pedestals of the TRD are determined every 5 minutes during the flight with a separate pedestal cycle. In flight, the pedestal variance in the TRD over that 5 minute period was typically $<1$ count.

\subsection{Trigger System}

Two classes of events are of interest for CREAM: high-energy shower events, regardless of the incident particle charge, and events with high-charge incident nuclei, whether or not they interacted and generated a shower in the calorimeter. The trigger system (Fig. 13), designed to initiate data collection for both is comprised of a Master Trigger, with inputs from the two detector trigger systems, calorimeter trigger and TCD trigger. The calorimeter trigger is designed for high energy shower events, while the TCD trigger is designed for events in which a high-charge nucleus traverses the TCD/TRD.

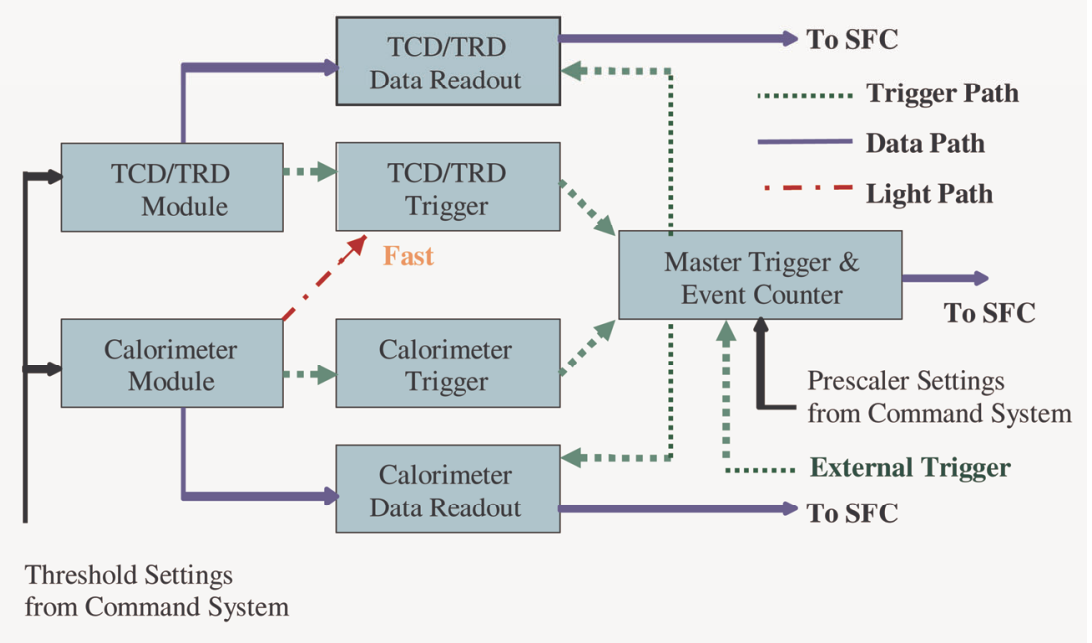

Figure 13. Trigger signal flow.

\subsubsection{Master Trigger}

The Master Trigger generates the signal initiating data collection from the sub-detectors and the building of this data into an event record. The Master Trigger accepts inputs from the Calibration System, calorimeter, and TCD. It provides 8-bit pre-scalers for the calorimeter and TCD to adjust the trigger rates in flight in steps of approximately $0.5 \%$, allowing separate control over the rate of each trigger type. Each valid trigger input produces trigger and event number outputs to each of the instrument subsystems, as well as trigger flags for the SFC to determine which trigger inputs are active. Each subsystem 
produces a data record for the SFC, which includes the event number. The SFC uses the event numbers to verify that all subsystems are providing data from the current event.

The Master Trigger electronics are designed with 74HC series high speed CMOS components, with a long history in the spacecraft industry. The trigger inputs and outputs are produced with LVDS (Low Voltage Differential Signaling) circuits, and distributed on twisted, shielded pair cables, to maintain fast signals with very low radiated emissions. The event number outputs use RS-422 circuits, which are distributed on twisted pair ribbon cables. The interface to the SFC consists of parallel I/O signals which are connected to a commercially available PC/104 board within the SFC.

\subsubsection{Calorimeter Trigger}

The calorimeter trigger, built around an Actel antifuse FPGA, requires $\mathrm{N}$ consecutive layers (with $\mathrm{N}$ set via the Command System to be 4, 6, 8, or 10) to be active to generate a high energy shower trigger. Simulation studies show the high energy shower trigger has an estimated efficiency above $90 \%$ for incident protons above $1 \mathrm{TeV}$, with a residual efficiency of about $20 \%$ at $100 \mathrm{GeV}$. However, the data rate after sparsification is low enough for the telemetry bandwidth, even with the high rate of lower energy particles. Further, this trigger model reduces biases to a minimum, allowing offline event selection to carefully remove background events. The calorimeter trigger board accepts the trigger signals generated from each of the calorimeter HPD boxes, and produces a trigger output to the Master Trigger whenever the selected consecutive layer requirement is met. The calorimeter HPD boxes provide 40 half-layer signals, which are combined to form 20 layer signals. A layer is considered active if at least one ribbon's low energy range (out of 50) registered a signal exceeding a commandable threshold, typically set at about $60 \mathrm{MeV}$. The rate for this trigger in flight ranged from $0.2 \mathrm{~Hz}$ to $3 \mathrm{~Hz}$, depending on altitude and threshold setting. In case a malfunction of some portion of the readout system disables the input from one or more layers, those layers can be bypassed (equivalent to "always on") to allow continued triggering. The calorimeter trigger accepts commands from the Command System to modify a "layer bypass mask" and/or modify the number of consecutive layers required for the high energy shower trigger.

\subsubsection{TCD Trigger}

The TCD trigger system uses a redundant set of coincidences between several PMT signals and the CD (Fig. 14). High-Z triggers are generated, at a rate between $10 \mathrm{~Hz}$ and $30 \mathrm{~Hz}$, when a particle with high enough charge and energy traverses the TCD and generates a signal in the CD (the threshold for this trigger was initially set for $Z \geq 3$, but was raised several times in flight to optimize for boron and carbon nuclei). When a particle of $Z \geq 1$ passes through the TCD, produces a sufficiently high signal in the S3 and a confirming signal from the calorimeter arrives within $100 \mu \mathrm{s}$, a Low-Z trigger flag is generated. The eight concentrators used to read out the scintillation counters are arranged such that each unit reads out two adjacent PMTs (each attached to one end of two neighboring scintillator paddles). A single side of the TCD is triggered by a logical OR of the four PMTs from that side. The trigger from each side of the instrument is sent to the TCD trigger board via a ribbon cable using LVDS logic. The Low-Z trigger flag is generated when a threshold is crossed on the anode signal of one or more PMTs on at least 3 sides of the TCD, and the same condition is met for at least one side of S3, and a confirming calorimeter trigger is generated within $100 \mu \mathrm{s}$. A High-Z trigger signal is generated when a threshold is exceeded on the dynode 11 signal of at least one PMT per side, again requiring at least a 3-side coincidence, and the CD signal exceeds a set threshold, indicating that the incident particle is relativistic. This last condition reduces the background from the plentiful low energy, high-charge nuclei expected above Antarctica, where the geo-magnetic cutoff is much lower than at equatorial latitudes. For ground tests with cosmic muons, the TCD trigger's High- $Z$ and Low- $Z$ outputs can be swapped to allow triggering on $Z=1$ particles that do not generate a shower in the calorimeter. 


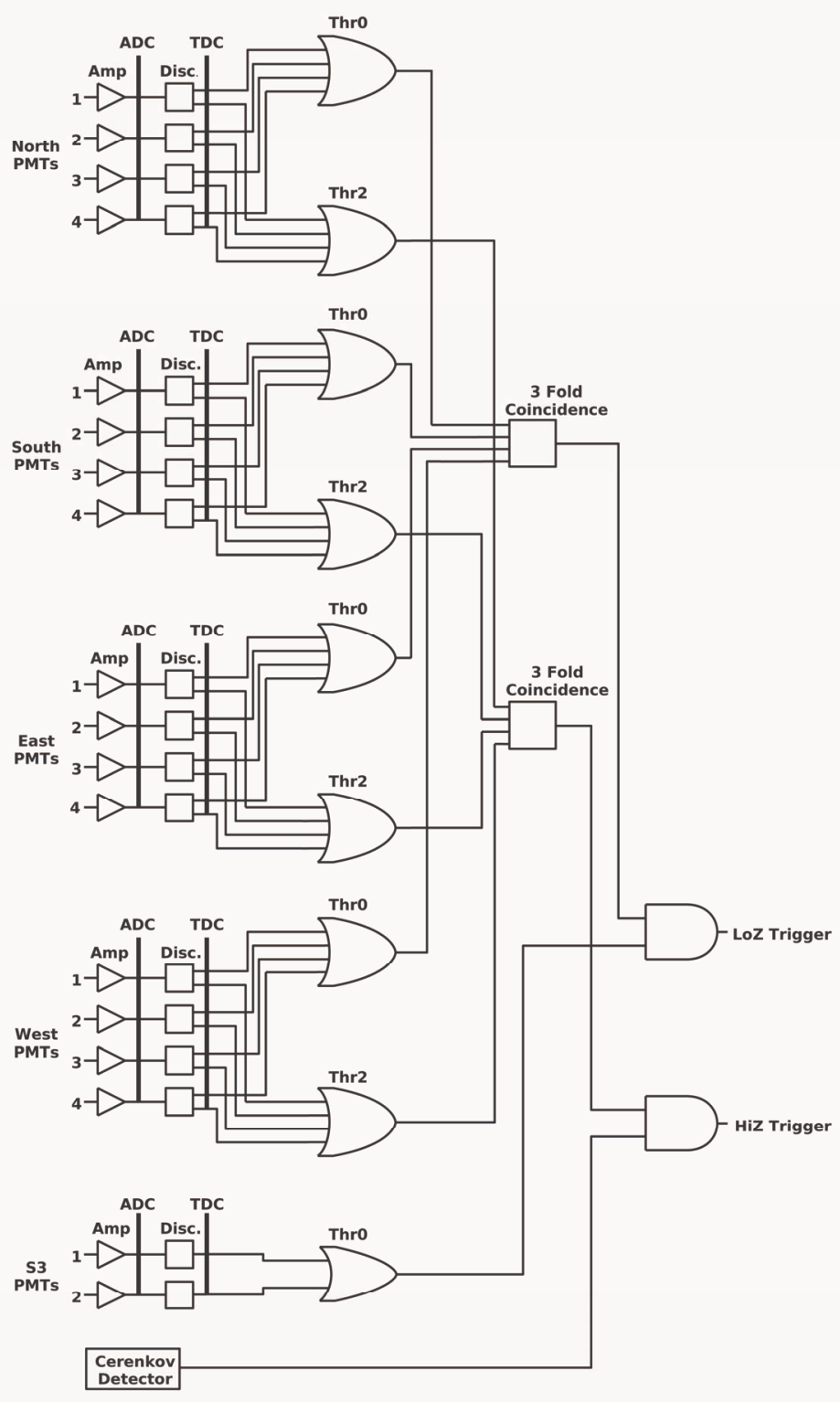

Figure 14. TCD trigger logic diagram.

\subsection{Command System}

The Command System is designed to allow individual commands and command scripts to be sent via NASA's Integrated Test and Operations System (ITOS) system. Instrument commands are entered via a text-based interface or through a graphical user interface (GUI) to a dedicated Linux workstation in the Science Operations Center (SOC) at the University of Maryland (UMD) from which they are sent to the Engineering Support Center (ESC) at NASA's Wallops Flight Facility (WFF) through the internet. The commands are forwarded to White Sands, NM to be sent up to the payload through TDRSS. Commands are received by the TDRSS transponder in the Command and Data Module (CDM) which checks if the commands are intended for its internal systems. If not, the commands are forwarded to the SFC, which 
determines if the commands are to be implemented internally, and if not, forwards them to the Command System's Atmel 89S8252 processor via a standard RS-232 serial port as ASCII characters. The processor formats the commands as appropriate, forwarding them to the correct box as RS-422 differential signals, and then sends an acknowledgement to the SFC. A reset signal is included in the command interface, which can be activated as a global reset or on a per box basis. When the balloon traverses a TDRSS zone of exclusion (ZOE) and none of the TDRS satellites is in view, a backup link using the Iridium satellite system can be used for command uplink and/or to down-link a reduced stream (up to $2.4 \mathrm{kbps}$ ) of housekeeping data. The Atmel 89S8252 processor is based on the Intel 8051 architecture, providing a reasonable instruction set, good bit-level instructions, and a wide variety of development tools. This device has the additional advantage of internal flash memory, which is in-circuit programmable through a simple serial interface. This quick re-programmability feature greatly reduces development and debugging effort. Redundant program memory is used to increase reliability by including a provision to switch the execution memory between internal flash and external erasable programmable read only memory EPROM (Erasable Programmable Read-Only Memory). This processor is readily available in industrial temperature range, and is operated at $3.7 \mathrm{MHz}$ to keep the power consumption very low.

\subsection{Housekeeping System}

The Housekeeping System monitors and periodically reports the readings of nearly 400 sensors and rates to monitor the health of the instrument. The system is built around the same Atmel 89S8252 processor used in the Command System. In this system too, a provision to switch the execution memory between internal flash and external EPROM improves reliability and the processor is operated at $3.7 \mathrm{MHz}$ to keep power consumption very low. The Housekeeping System has 256 individual analog inputs with some channels sub-multiplexed and synchronized with RS-422 control signals to acquire 130 multiplexed analog signals from the SCD. Housekeeping requests and returned data are communicated to the SFC via a standard RS-232 serial port as ASCII characters. Reporting frequency can be changed by command, and was typically set to send a packet every 5 to 10 seconds in flight. The system monitors a total of 96 temperatures, 85 voltage levels, 149 current levels and 12 pressure levels. In addition, housekeeping packets report the rates of each trigger type from the Master Trigger, the rates of the 40 individual calorimeter half-layer trigger signals, GPS location from the CDM, the remaining free space on the flash disk (Section 3.12.1), and the levels of several data buffers in the SFC. Where appropriate (e.g. power consumption, temperature, voltage and/or currents) each level being monitored is compared to "yellow" and "red" limits to alert the science shift operator to any incipient problems and emergency situations, respectively. By monitoring for changes following commands, the operator is able to use the Housekeeping System to verify command execution. The Housekeeping System also includes a pair of 48-bit counters to keep track of cumulative total time and live time.

\subsection{Calibration System}

To allow correct interpretation of flight data, calibration systems are implemented for the different detector systems. These use a variety of means to check aliveness, measure the noise in the absence of a physics signal (pedestal values), and determine the gain of each channel. The TRD and CD use science event data for calibration, so no dedicated calibration system is implemented for these.

\subsubsection{Calorimeter and Hodoscope Calibration System}

The calorimeter/hodoscope Calibration System is designed to provide triggers and voltage pulses to collect pedestal, charge injection and LED flasher data. The rates, pulse amplitude, and pulse width are all adjustable by command. The frequency is selectable from $1 \mathrm{kHz}$ down to about $0.06 \mathrm{~Hz}$ in multiples of 2. Charge calibration pulses from 0 to $2 \mathrm{~V}$ are provided by 24 pulsers with 14-bit resolution. Light pulses are provided by 8 LEDs driven by voltages from 0 to $8 \mathrm{~V}$ with 14-bit resolution. The pulse widths 
are adjustable from $\sim 600 \mathrm{~ns}$ to $5 \mu \mathrm{s}$. Pedestal runs were collected every 5 minutes in flight to account for pedestal drift of 12 (12-bit) ADC counts per degree $\mathrm{C}$ for the calorimeter and 18 (16-bit) ADC counts per degree $\mathrm{C}$ for the hodoscope, during the diurnal cycle. The mean value and RMS width of the pedestal are calculated for each channel and used to update the sparsification threshold values using the selected sparsification algorithms. The pedestal values are included in the data stream to allow offline subtraction of the mean offset level of each channel. Periodic runs of charge injection events are collected and included in the data stream to allow offline gain calibration of the calorimeter and hodoscope data. The LED system is used during integration and testing to align the HPD pixels with the fibers carrying the light signal from the detectors. This is done by rotating and moving either the cookies holding the fibers (calorimeter) or the HPD boxes (hodoscopes) while flashing the LEDs, and monitoring cross-talk in the channels surrounding the LED channels. Once the cross-talk around the LEDs is balanced, the alignment is deemed to be done. In flight, the LEDs were used on occasion to check the aliveness of HPDs. The calorimeter and hodoscope data is compared to sparsification thresholds (Section 3.7) updated based on the constantly revised pedestal levels. This sparsification is applied to all but 40 calorimeter and 36 hodoscope channels (one per HPD) to allow removal of coherent noise.

\subsubsection{SCD Calibration System}

The SCD Calibration System is designed to provide charge injection pulses up to $3.2 \mathrm{pC}$ into the SCD readout system to monitor gains. The system can be used to collect data with no charge input to monitor pedestal levels. In flight, pedestals were collected every 5 minutes to allow accurate pedestal subtraction in offline data analysis, as pedestal variations due to temperature are about 20 (16-bit) ADC counts per degree $\mathrm{C}$ for most channels. The diurnal variation in sun angle changes the solar flux on the payload, causing the SCD temperature to vary within a $4 \mathrm{C}$ range, translating to an $80 \mathrm{ADC}$ count range. Charge calibration was carried out every 2 hours, showing stable gain throughout the flight. The SCD data is compared to sparsification thresholds (Section 3.7) that are updated based on the constantly revised pedestal levels. Sparsification is applied to all but 26 SCD channels (one per ladder) to allow removal of coherent noise.

\subsubsection{TCD Calibration System}

Before flight, the high voltage to each PMT is set to provide a clear muon peak in the ADC output, well separated from the pedestal. Using information from the CERN beam tests (Section 4.1.3), the TDC values are set to provide the Low- $\mathrm{Z}$ and High- $\mathrm{Z}$ triggers described in Section 3.8.3. Flight data were also examined in near real-time to ensure that signals due to helium nuclei did not "leak into" the High-Z trigger. The four ADC channels per PMT are designed to overlap for a portion of their sensitive range, allowing flight data to be used for inter-calibration of the gains.

\subsection{SFC}

The instrument is operated and monitored via a central computer, the SFC. The SFC builds data from the different detector subsystems into a single event record each time the Master Trigger signals a valid event has occurred. The SFC then splits the event record into packets that it sends to the CDM for archival and telemetry to the ground. Hardware commands are passed through the CDM and SFC to the various modules, with software commands modifying how the SFC operates.

\subsubsection{SFC Hardware}

The SFC is based on a single-board computer with a PC/104 interface. This architecture is chosen based on past experience of the Columbia Scientific Ballooning Facility's (CSBF) Science Interface Package 
(SIP) and the NASA/WFF CDM. It has the advantages of low power consumption and a simple bus interface for instrument subsystems to integrate with. Although thermal strapping is required on the computer board, it is relatively easy to build interfaces for this bus that dissipate low enough power to avoid the need for any special thermal provisions.

Each instrument subsystem has its own PC/104 interface card, with the exception of the TCD which interfaces via Ethernet, and the Command and Housekeeping modules, both with low enough bandwidths to be accommodated with standard serial ports. The calorimeter, hodoscopes and SCD each use an identical custom-designed interface, with sparsification thresholds stored in a dual-port RAM, and a PC/104 interface implemented in an Actel antifuse FPGA. The TRD uses a commercially available PC/104 board, with a PC/104 interface implemented in a Xilinx Spartan-2 FPGA. The Master Trigger uses a commercially available parallel interface card, with additional I/O capacity for various single bit control functions. To provide additional archiving of lower priority data not expected to be sent via telemetry, the SFC also has a 43 GB BitMicro E-Disk EIDE solid-state flash drive, mounted on top of the SFC box. Data from this archive are merged post-flight with telemetered data and those archived in the CDM to build up the complete set of flight data.

\subsubsection{SFC Operating System}

The SFC utilizes a RedHat 7.3-based Linux operating system, stripped of unused components, and consisting of the kernel and the utilities to allow self-hosted development. Real-time extensions to the Linux kernel were evaluated, but were not needed to maintain the required data-flow or interrupt handling. The operating system choice was frozen early in the payload development as stability was deemed significantly more important than software upgrades. The flight operating system, utilities, and flight software all fit in $256 \mathrm{MB}$. The operating system and flash drive worked well during the nearly 42 day flight, though the Ethernet switch connecting the TCD, SFC, and CDM did require several reboots. This latch-up behavior was not observed on the ground and may have been due to single-event upsets at float altitude.

\subsubsection{CREAM Data Acquisition (CDAQ) Software and Telemetry}

The CDAQ $[21,22]$ is required to provide a very robust tool for processing commands from the ground through the CDM, and for consolidating science, calibration and housekeeping events, transferring this data to the CDM with the necessary error-checking information. As such, the CDAQ is required to initiate and collect scheduled calibration and housekeeping events, but at the same time react quickly whenever a command is received from a CDM computer, or a trigger signal is issued by the Master Trigger. The CDAQ has limited hardware resources given the power and interface constraints that determine the hardware, and as such its design principles are simplicity, reliability, and flexibility. A server/client model is adopted as best suited for telemetry of commands and data, with the ground operations center software set up as the client, and the SFC set up as the server. Wherever possible, the number of processes active on the SFC is minimized, with a master process managing all other processes. Circular ring buffers based on shared memory with semaphore locks allow processes to pass commands or event data efficiently. Threading and real-time schemes are adopted for communications with the CDM to allow efficient and stable long-term function. The communications process also splits up event records into packets, adding packet management and checksum bits. These packets are further processed by the CDM, adding another layer of packet management and checksums, and are sent to the ground via the TDRSS high gain antenna. To maximize telemetry efficiency, event records are tightly packed and allowed to cross packet boundaries. On the ground, the process is reversed, with the computers at the ESC reconstructing the data to match what the CDM received from the SFC and sending it via the internet to the SOC at the University of Maryland. There, ground operations computers reconstruct events from the packets received from the ESC, with a fraction of science events passed to an event monitoring 
software, and all housekeeping records updating a housekeeping viewer. Whenever CREAM is in a TDRSS ZOE, the CDM archives data, playing it back upon the payload's exiting the ZOE. Such playback data are recorded to disk but are not used to update any online monitoring, as they are superceded by later data. Separate processes handle housekeeping, calibration, and event data so that failure of one process does not interfere with the function of the others. The event data process constantly monitors the Master Trigger and builds events whenever a trigger signal is issued. Ground operations machines run remote commanding and GUI processes to allow operators to issue commands to the instrument systems. To make operations more efficient and reliable, predefined scripts are stored on the ground operations machines for routine procedures that require dozens of individual commands (e.g. turning on boxes, starting high voltage ramping, etc.).

Communication between the SFC and the CDM computer is via a custom UDP (User Datagram Protocol)

Ethernet protocol. Being connectionless, this protocol makes it easier to re-establish communication if one of the sides loses power temporarily. Information is passed via fixed length packets, with connection status packets exchanged in both directions every five seconds asynchronously. This allows the SFC to determine which of the redundant CDM computers is active and what port it is on, so that the address can be modified whenever a change is detected (only the SFC UDP port number is specified in advance). Event and housekeeping data is passed via 255 byte packets, with 5 of those bytes providing packet management. Since event record sizes depend on the physics of the event and the settings of the Sparsification System, ranging from 4 bytes to $64 \mathrm{kB}$, a packet can hold dozens of event records, or be a small fraction of an event record. Whenever the SFC sends a data packet, the CDM computer is expected to send back an acknowledgement packet. Lacking this acknowledgment, the SFC will repeatedly send the same packet every second until it is acknowledged or 30 attempts have been made. If no acknowledgement is received after 30 attempts, the SFC drops the connection, pings both CDM computers, and re-establishes the connection. Once communication resumes, the SFC starts over with the same packet it attempted to send before. To avoid an infinite loop, a packet will ultimately time out, and the SFC will proceed to the next packet. In flight, nearly $100 \%$ of packets were acknowledged without retry. Whenever the SFC receives a command packet from the CDM, it checks a CRC2 (Cyclic Redundancy Check) checksum and sends an acknowledgement packet. Command packets can be either an 8 byte command or a 1050 byte fragment of an extended command. The SFC communications process reassembles the 8 fragments of an extended command, providing the capability of e.g. updating sparsification threshold value tables, transferring files to the SFC, etc. Since satellite links occasionally corrupt packets, the SFC generates a 16-bit CRC for each packet, and includes the least significant bit in the packet (one of the 5 packet management bits). Such corruption was experienced in flight mostly during entry into or exit out of a ZOE. The error checking was nearly perfect, and in fact only 29 telemetered events were corrupted. This constitutes an error rate of less than $10^{-6}$ of events, or $10^{-10}$ bits, compared to the requirement of $10^{-6}$ bits. These events were later recovered from the CDM archive disks.

\subsection{Power System}

Fused +31 VDC power is provided by the balloon-craft power system inside the CDM to six separate circuits, with separate relays. These power the calorimeter power converter, the hodoscope power converter, the SCD power converter, the TCD power converter, the TRD power converter and the SFC, with a total of $379 \mathrm{~W}$.

\subsubsection{Calorimeter Module Power Systems}

The power systems for the calorimeter, hodoscopes, and SCD each use three Vicor VI-J00 series DC/DC converters with VI-RAM active output filter modules, to convert the $28 \pm 5 \mathrm{~V}$ input from the balloon-craft main power bus to the voltages needed to power their respective detector systems (calorimeter $\pm 3.5 \mathrm{~V} \&$ 
$12 \mathrm{~V}$; hodoscopes $\pm 6 \mathrm{~V} \& 12 \mathrm{~V}$; SCD $\pm 6.5 \mathrm{~V} \& 12 \mathrm{~V}$ ), with a total power of $185 \mathrm{~W}$. Common-mode choke filtering is used to reduce the conducted emissions on the power system input bus to acceptable levels. A power control board, mounted over the converters, provides power switching for the control of the three voltages distributed to the 14 individual boxes. High-side current monitoring is provided for each voltage distributed to each box. The monitored currents (represented by a voltage) are sent to the Housekeeping System. The three primary voltage levels are also sent to the Housekeeping System for monitoring. The control board accepts commands from the Command System to turn on or off the power to each of the individual boxes through logic circuits. An over-current shutdown function using comparators to compare to a set of thresholds (set by resistor selection) allows switching circuits off through the same logic circuits when needed. This protection feature includes a delay override to prevent spurious shutdown due to fast transient spikes at turn-on or from noise. A power-on reset circuit ensures that all power to each individual box is switched off when the logic circuits are first powered on.

\subsubsection{TCD Power System}

The TCD power system mounted on the pallet supplies the various regulated voltages $(-2 \mathrm{~V},+3.3 \mathrm{~V}, \pm 5 \mathrm{~V}$ $\&+12 \mathrm{~V}$ ) needed by the TCD system, with a total power of $110 \mathrm{~W}$. Each voltage is provided by a circuit board consisting of an industrial grade Vicor DC-DC converter module and a Vicor ripple attenuation module from the same series as used in the calorimeter module power systems. The input to each board also contains a resettable fuse and an LC filter.

\subsubsection{TRD \& CD Power System}

The TRD and CD power systems are based on Vicor VI- 200 series converters providing $+5 \mathrm{~V} \& \pm 12 \mathrm{~V}$ from the main balloon-craft power bus. These Vicor supplies have been used successfully on many previous balloon payloads and have a high efficiency (above 90\% at full load). The TRD/CD power system is designed to keep power consumption as low as possible. The overall power consumed by the TRD is $\sim 37 \mathrm{~W}$ while the power for the Cherenkov Detector is $\sim 10 \mathrm{~W}$.

\subsubsection{SFC Power System}

A commercial wide-temperature range $\mathrm{PC} / 104$ power supply is used to convert the main balloon-craft power bus $31 \mathrm{VDC}$ to $+5 \mathrm{~V}$ and $+12 \mathrm{~V}$ for computer use. The SFC Power System also supplies power to the Master Trigger and Housekeeping modules.

\subsection{Weight and Power}

Two major considerations in balloon experiments are the limited weight the balloon can carry to float altitude, and the limited power that can be provided by either batteries or solar arrays. The CREAM instrument is designed to optimize both the weight and power while collecting high quality data at as high a rate as possible. The 40 million cubic foot "light" zero pressure balloon used by NASA allows a suspended weight of up to $2727 \mathrm{~kg}$. After considering the flight train (cables, parachute, etc.), support systems (power, telemetry, rotator, crush pads, etc.), an instrument weight of about $1143 \mathrm{~kg}$ (Table 1) allows $500 \mathrm{~kg}$ of ballast. Such a relatively large ballast weight makes extended flight durations more likely. The power system is designed to provide sufficient power for extended flights, where the sun angle could dip low, reducing the solar flux and the generated power. With a 10 panel solar array, the power system was able to generate about $1400 \mathrm{~W}$ at altitude during the first flight (see below). During the first few days, about $200 \mathrm{~W}$ of excess power was routed to shunt resistors mounted under the legs of the payload where it was radiated away. As the flight continued, less excess power was generated. The support systems use an average of about $400 \mathrm{~W}$, with another $379 \mathrm{~W}$ used by the instrument (Table 1). The dimensions in Table 1 are general ones, with the total size being the lateral size of the pallet 
constituting the base of the instrument support structure, and the total height measured from the top surface of the pallet to the top of the uppermost detector - the TCD.

\begin{tabular}{|l|c|c|c|}
\hline \multicolumn{1}{|c|}{ Detector System } & Weight & Power & Dimensions \\
\hline Upper detectors (TRD, CD, TCD) & $396 \mathrm{~kg}$ & $159 \mathrm{~W}$ & $125 \times 125 \times 94 \mathrm{~cm}^{3}$ \\
\hline Targets \& interleaved detectors (SCD, hodoscopes) & $219 \mathrm{~kg}$ & $131 \mathrm{~W}$ & $80 \times 80 \times 24 \mathrm{~cm}^{3}$ \\
\hline Calorimeter & $378 \mathrm{~kg}$ & $54 \mathrm{~W}$ & $50 \times 50 \times 10 \mathrm{~cm}^{3}$ \\
\hline Common electronics & $150 \mathrm{~kg}$ & $35 \mathrm{~W}$ & $\mathrm{~N} / \mathrm{A}$ \\
\hline Total & $\mathbf{1 1 4 3} \mathbf{~ k g}$ & $\mathbf{3 7 9} \mathbf{W}$ & $\mathbf{1 8 0} \times \mathbf{1 8 0} \times \mathbf{1 2 8} \mathbf{~ c m}^{\mathbf{3}}$ \\
\hline
\end{tabular}

Table 1. Weight, power and dimensions of instrument subsystems.

\section{BEAM TEST and FIRST FLIGHT}

\subsection{Beam Tests}

Over the course of the 3 years prior to the first flight, various sub-detectors were exposed to high energy particle beams at CERN's Super Proton Synchrotron (SPS) facility. In 2001 a partially populated full depth calorimeter was tested, along with a one-layer prototype of the TRD. In 2002 a fully instrumented calorimeter was tested to verify its performance, along with a prototype hodoscope. A prototype SCD sensor was also exposed that year to validate the design and performance. In 2003, a TCD paddle was tested along with the hodoscopes (with partial readout). In parallel, the fully instrumented calorimeter was calibrated. In late 2003, a crossed pair of TCD paddles (with prototype readout electronics), the calorimeter, hodoscopes, and SCD were all exposed to a beam of $\mathrm{A} / \mathrm{Z}=2$ fragmentation products from an indium beam with $158 \mathrm{GeV} / \mathrm{n}$ momentum. With these beam runs, various detector system designs were validated, and where needed, design improvements were implemented.

\subsubsection{Validating and Calibrating the Calorimeter}

To calibrate the calorimeter one needs to deposit known amounts of energy in each of the 1000 fiber ribbons. Given sufficiently high signals, it is reasonably simple to equalize the response from the 50 ribbons in a given layer, by moving the detector in steps of $1 \mathrm{~cm}$ vertically or $1 \mathrm{~cm}$ horizontally, such that a high energy electron beam is centered each time on the center of a different ribbon in each X or Y layer. Using Monte Carlo simulations of electron showers it is also possible to calculate the energy deposit expected along the shower core in each layer. This allows one to equalize the ribbon responses between layers. The calorimeter is designed to measure the energy deposit from showers initiated by nuclei with energies up to $10^{15} \mathrm{eV}$ and higher. This is a sampling calorimeter with a sampling fraction of about $0.3 \%$ for minimum ionizing particles, where isotropically incident $\mathrm{TeV}$ proton showers initiated in the graphite targets deposit about $0.2 \%$ of their incident energy in the active media. With such low sampling, and with electron test beam energies of $150 \mathrm{GeV}$ or less, only 8 to 10 layers around the shower maximum register enough scintillation to allow calibration. To address this, the calibration scan was carried out in three sets of runs. In the first set, intended to calibrate the center 10 layers, the calorimeter and graphite targets were positioned horizontally in the beam, simulating vertically incident particles. In the second set, 5 radiation lengths of lead were placed in the beam immediately before the graphite targets, thereby moving the shower maximum up and allowing the first 4 layers to be calibrated. Following that, the experiment was rotated such that the beam was incident through the aluminum honeycomb pallet and the bottom of the calorimeter, to calibrate the bottom 4 layers. In this orientation, the same 5 radiation lengths of lead used in the second run would be insufficient, since unlike the top of the calorimeter, where a tungsten plate precedes the first active layer, the bottom calorimeter plate is made of aluminum. In addition, the 1 radiation length target in this configuration is behind the calorimeter. Thus 2 radiation lengths of tungsten were placed in the beam along with the 5 radiation lengths of lead, both just in front of the pallet bottom 
surface. Following this calibration, the data were compared to simulated $150 \mathrm{GeV}$ electrons (Fig. 15) showing excellent agreement [23]. Inter-calibrating between the low- and mid-energy ranges, and between the mid- and high-energy ranges requires very high energy particles, which are beyond the range of test beams available at this time. However, this inter-calibration can be carried out with flight data by comparing the signals from two ranges of the same ribbon generated by the same shower. Using only those cases where both ranges register significant signals, but where neither range saturates, one can extract the gain constant between the two ranges.

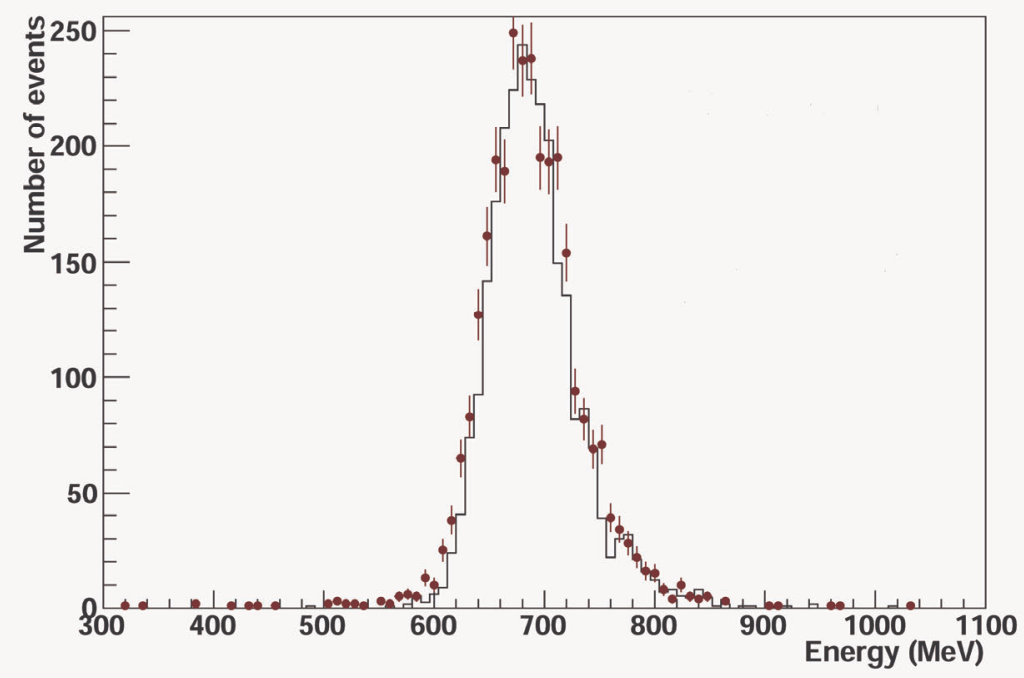

Figure 15. Energy deposited in calorimeter by $150 \mathrm{GeV}$ electrons - data (markers) and simulation (histogram).

\subsubsection{Validating the SCD}

In November 2003 the SCD was exposed to a beam of $(\mathrm{A} / \mathrm{Z}=2)$ nuclear fragments generated from a 158 $\mathrm{GeV} / \mathrm{n}$ indium beam incident on a thin target at CERN [24]. The beam was collimated to hit only one pixel at a time (less than $2 \mathrm{~cm}^{2}$ ), and the SCD was moved up/down and left/right with respect to the beam to expose different sensors to the beam. The results (Figs. 16, 17) show clear separation of all peaks from $Z=2$ to well above $Z=28$, with a charge resolution of $<0.2 \mathrm{e}$ based on a Landau fit of the He data.
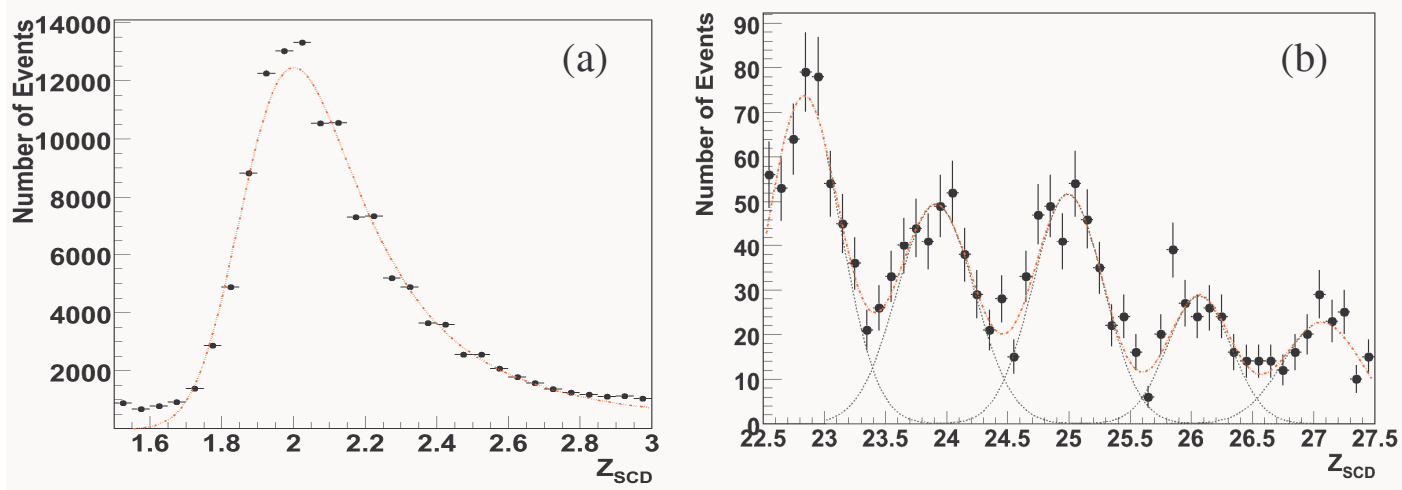

Figure 16: Distribution and Landau fit of measured SCD charge for events from indium beam fragments at CERN with $\mathrm{Z}=2$ (a) and $23 \leq \mathrm{Z} \leq 27$ (b). The resolution achieved exceeds the required $0.2 \mathrm{e}$ for helium and $0.3 \mathrm{e}$ for Fe. 


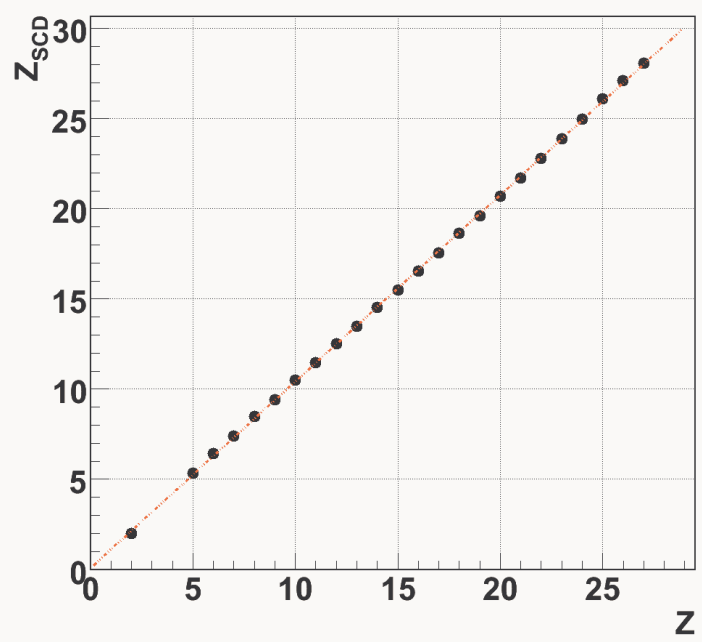

Figure 17. SCD reconstructed charge vs. actual charge showing excellent linearity beyond $Z=26$.

\subsubsection{Validating the TCD}

Components of the TCD were tested at CERN in the same A/Z = 2 beam as the SCD. The TCD components in the test consisted of two crossed scintillation counters and prototype flight electronics, including a dedicated computer running $\mu$ Clinux, a trigger board, four TDC boards, and two peak detector (ADC) boards. This allowed each tube to be instrumented with two TDCs and two ADCs, all read out via Ethernet. Excellent charge resolution was obtained from the ADC readouts, ranging from about 0.2e for O nuclei to about 0.35e for Fe nuclei, as shown in Fig. 18.

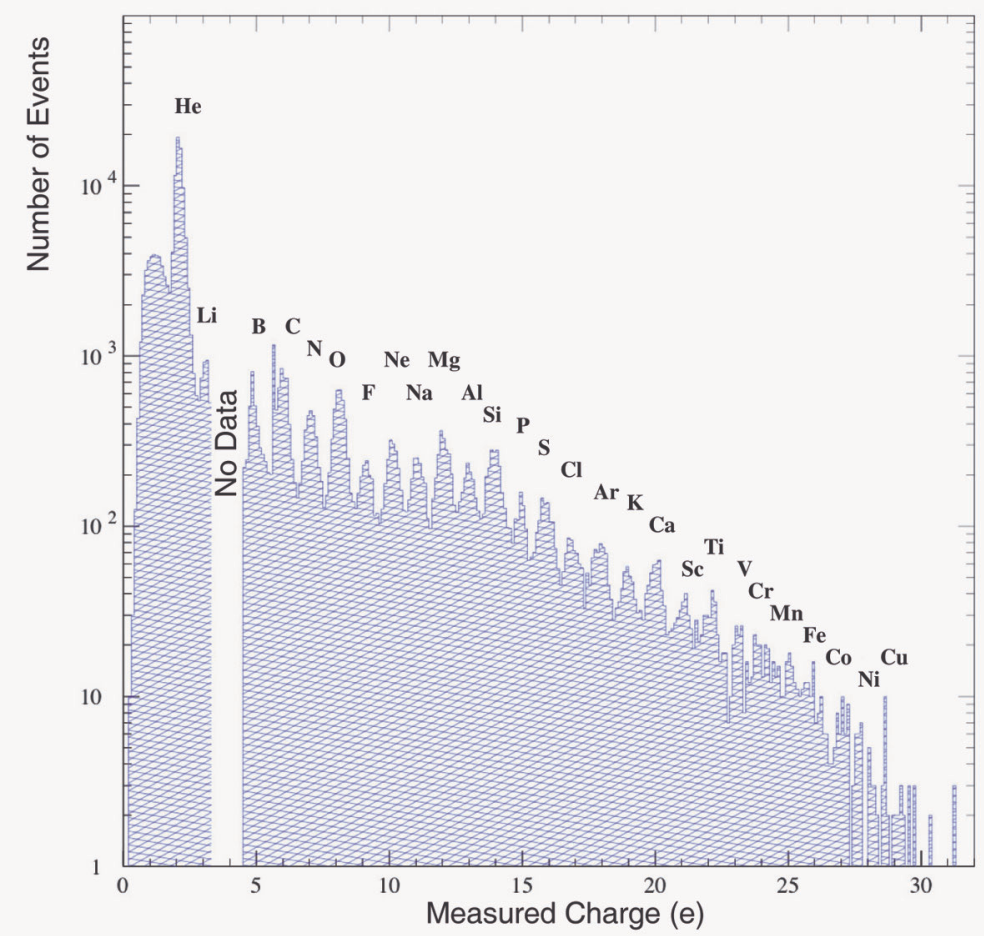

Figure 18: Distribution of measured TCD charge for events from $A / Z=2$ nuclear fragments at CERN. 


\subsubsection{Validating the TRD}

A prototype of the TRD was exposed to accelerator beams at CERN in 2001. Part of the purpose of this calibration was to optimize the foam material used as a radiator upstream on the xenon-filled tubes. The combination of tube, gas and wire parameters provides a signal clearing time of $\sim 500 \mathrm{~ns}$ well matched to the Amplex 1.5 ASIC shaping time. For the beam test an auxiliary tracking detector was built to identify the particle location with an accuracy of $\sim 100 \mu \mathrm{m}$ using two small multi-wire chambers with charge division readout in the direction perpendicular to the sense wires. These were instrumented with the same Amplex-based systems used for the TRD tubes. The assembly was exposed to a range of beam momenta, and the calorimeter could be used for particle tagging. The Lorentz factors associated with electrons, muons, pions and protons were used to find the response over a range of values. In addition, the plastic foam radiator was removed from the test setup for some of the runs and replaced with an equivalent thickness of solid plastic to provide a "no-TR" response. The results of these measurements are shown in Fig. 19, where they are used to confirm a GEANT4 TR simulation of the device (upper and lower curves). The markers show measurements for protons (squares), muons/pions (inverted triangles) and electrons (triangles). The upper curve, representing the Ethafoam configuration ultimately used in the flight TRD, shows that the TR signal becomes significant above Lorentz factors of $\sim 1000$. The lower curve, representing the solid plastic configuration, shows the "no-TR" response, consisting essentially of the relativistic rise of ionization in the xenon gas.

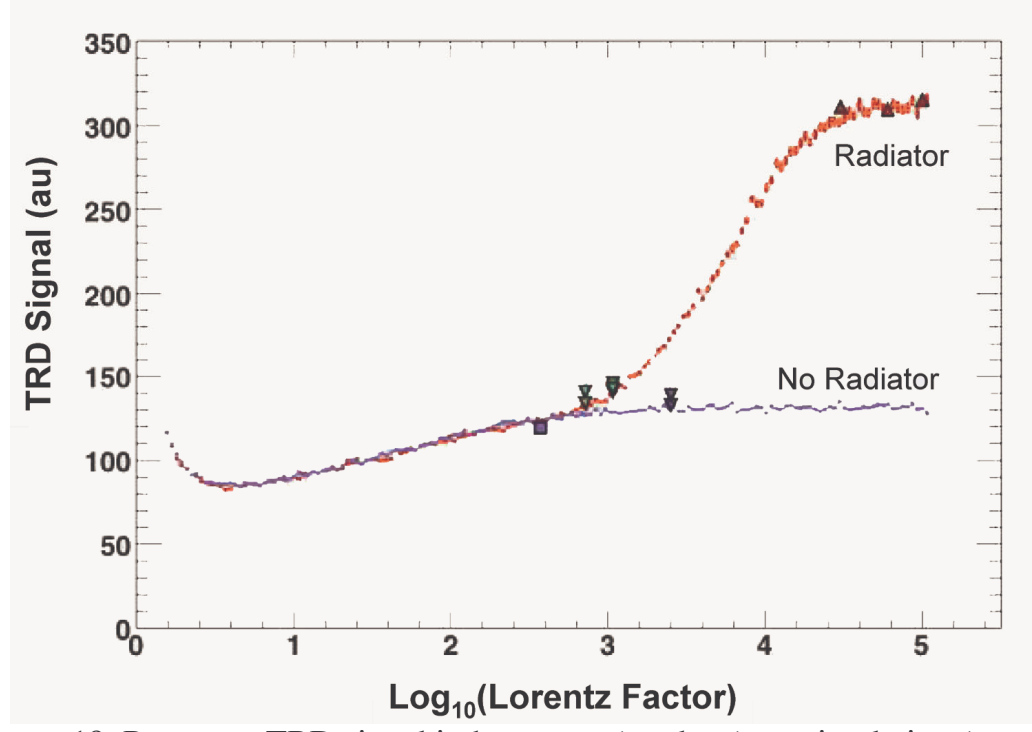

Figure 19. Prototype TRD signal in beam test (markers) vs. simulation (curves).

\subsection{First LDB Flight}

CREAM was first launched from Williams Field, outside McMurdo Station Antarctica on December 16, 2004, carried by a zero-pressure balloon and remained aloft for a record breaking 41 days 21 hours and 36 minutes, circumnavigating the South Pole three times. During this flight CREAM recorded 48.9 GB of data, including over 43,000,000 high-Z events, over 500,000 high energy shower events (e.g. Fig. 20) with several above $100 \mathrm{TeV}$, and over 2,000,000 calibration and housekeeping records. The detector performed as expected, and preliminary data analysis shows great promise [25 and references therein]. A second CREAM flight was launched a year later to the day collecting data for nearly 28 days. In both flights data were received, stored and a subset was processed for quick-look analysis at the SOC. The SOC monitored the health of the instrument through housekeeping sensor data and generated the commands necessary to trim and maintain optimum science output from the package. 


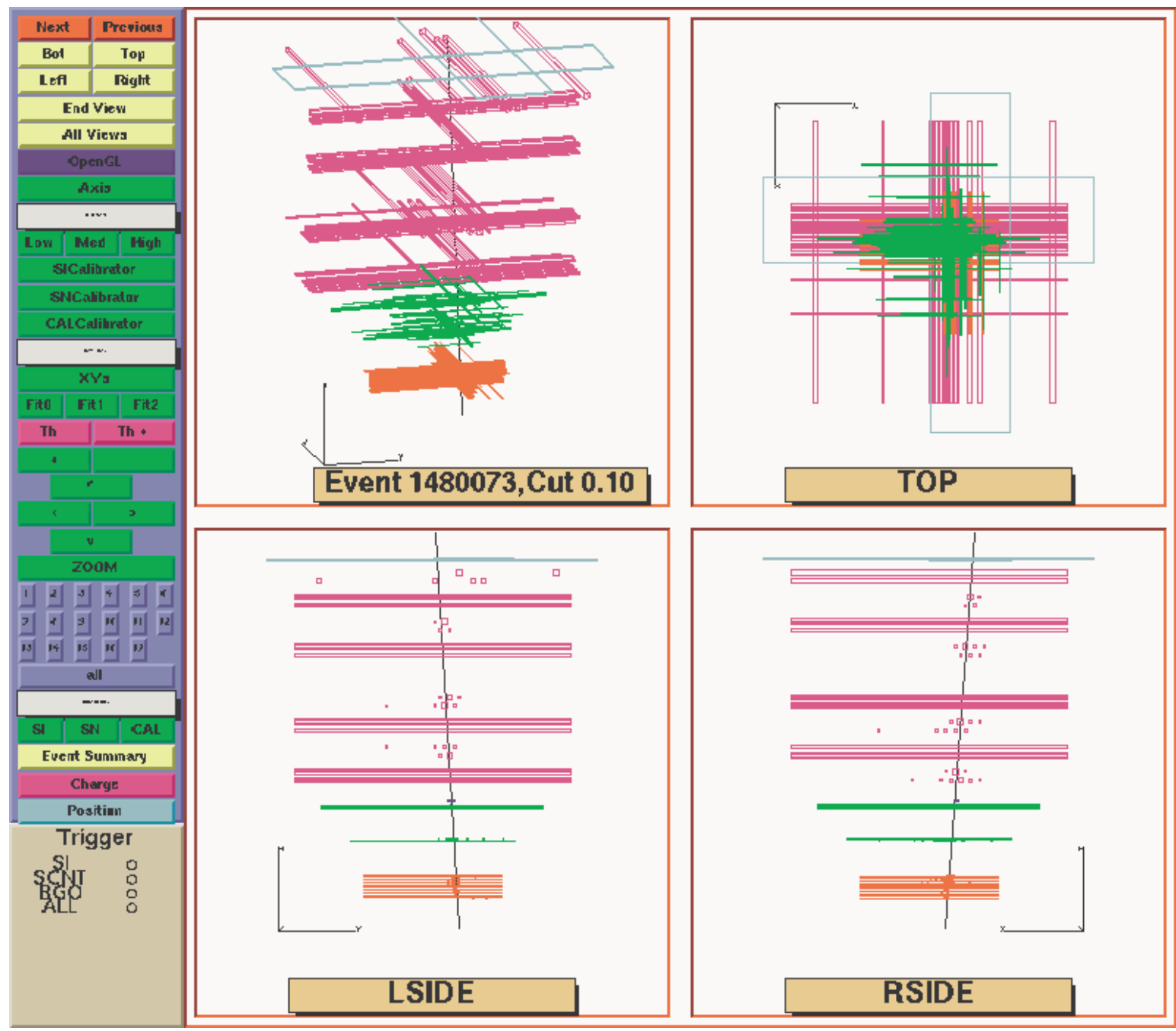

Figure 20. Online monitoring Event Display of a typical high energy shower event from the 2004/05 flight.

\section{CONCLUSIONS}

The CREAM experiment was designed to fly as a balloon payload under NASA science balloons (LDB and ULDB), directly measuring high energy cosmic-ray nuclei from hydrogen to iron, in the energy range from about $1 \mathrm{TeV}$ to over $1000 \mathrm{TeV}$. The experiment was optimized to enable as large a geometric factor and flight duration as possible given the constraints of the available balloon technology. CREAM is unique in that it employs redundant measurements of both energy and charge of the incident nuclei. As part of the extensive development program, the CREAM sub-detectors were tested and calibrated at CERN's SPS accelerator facility, proving that the detectors work as designed, and showing that the simulation correctly describes them, allowing extrapolation to the energy range of interest. With a total of 70 days flight duration for the two flights [26], the CREAM program now has produced what is arguably the largest and most exciting set of data for directly measured high energy cosmic-ray nuclei, with energies as low as $10 \mathrm{GeV}$ and up to well over $100 \mathrm{TeV}$. With further flights planned for these detector suites after refurbishment, the CREAM data set is expected to grow even more interesting in the coming years. 


\section{ACKNOWLEDGMENTS}

The work reported in this paper was funded by NASA research grants to the University of Maryland, the University of Chicago, Penn State University, and the Ohio State University, by the Korean Ministry of Science and Technology in Korea, and by INFN in Italy. The authors wish to acknowledge NASA/WFF for provision and operation of flight support systems; Art Ruitberg, Suong Le, and Curtis Dunsmore of NASA/GSFC, and Carlos Urdiales of Southwest Research Institute for assistance with HV design and potting; CERN for provision of excellent accelerator beams; the Fermi National Accelerator Lab Thin Films Group for high quality polishing and aluminization of optical elements; and Columbia Scientific Ballooning Facility, National Science Foundation's Office of Polar Programs, and Raytheon Polar Services Company for outstanding support of launch, flight and recovery operations in Antarctica. The TCD group acknowledges engineering contributions made by L. Engel, J. Passaneau and S. Posey. The

TRD group acknowledges engineering contributions made by R. Northrop and G.A. Kelderhouse. The SCD group acknowledges contributions made by W. Han, H.J. Hyun, H.J. Kim, M.Y. Kim, K.W. Min, H. Park and K.I. Seon. The S2 group acknowledges engineering contributions made by A. Basti.

\section{BIBLIOGRAPHY}

[1] P.O. Lagage \& C.J. Cesarsky: Astronomy \& Astrophysics 118, (1983) 223.

[2] H. Schieler et al. (KASKADE Collaboration), Proc. of SPIE, Particle Astrophysics Instrumentation, Vol. 4858, (2003) 41.

[3] T. Antoni et al. (KASKADE Collaboration): Astroparticle Physics 24, (2005) 1.

[4] T.G. Guzik et al. (ATIC Collaboration), The ATIC long duration balloon project, in The Next Generation in Scientific Ballooning, Advances in Space Research 33/10, (2004) 1763.

[5] K. Asakimori et al. (JACEE Collaboration), Cosmic Ray Proton and Helium Spectra - Results from the JACEE Experiment, Astrophysical Journal 502, (1998) 278.

[6] V.A. Derbina et al. (RUNJOB Collaboration), Cosmic-ray spectra and composition in the energy range of 10-1000 TeV per particle obtained by the RUNJOB experiment, Astrophysical Journal 628, (2005) L41.

[7] D. Müller et al. (TRACER Collaboration), Measurements with TRACER: discussion of results and future prospects, Proc. $29^{\text {th }}$ ICRC, Pune, India, 3, (2005) 89.

[8] S.P. Wakely et al. (CREAM Collaboration), First measurements of secondary nuclei at high energy with CREAM, Advances in Space Research, (2007) in press.

[9] O. Ganel et al. (CREAM Collaboration), Cosmic Ray Energetics And Mass (CREAM): Calibrating a Cosmic Ray Calorimeter, Proc. $10^{\text {th }}$ International Conference on Calorimetry in Particle Physics, Pasadena, CA, (2002) 133.

[10] O. Ganel et al., A Guide for ACCESS Design Considerations, Proc. STAIF - 1999, AIP Vol. 458, (1999) 272.

[11] E.S. Seo et al. (ATIC collaboration), The Advanced Thin Ionization Calorimeter (ATIC) Experiment: Expected Performance, in Gamma-Ray and Cosmic Ray detectors, Techniques, and Missions, Proc. SPIE International Symposium on Optical Science, Engineering, and Instrumentation, Vol. 2806, (1996) 134.

[12] J.J. Beatty et al. (CREAM collaboration), The Cosmic Ray Energetics and Mass (CREAM) Experiment Timing Charge Detector, Proc. SPIE, Particle Astrophysics Instrumentation, Vol. 4858, (2003) 248.

[13] E.S. Seo et al. (CREAM Collaboration), Cosmic-ray energetics and mass (CREAM) balloon project, Advances in Space Research 33/10, (2004) 1777.

[14] P.J. Boyle, S.P. Swordy and S.P. Wakely, Comparison of a transition radiation detector response with numerical simulations, Proc. $28^{\text {th }}$ ICRC, Tsukuba, Japan, (2003) 2233.

[15] I.H. Park et al. (CREAM collaboration), Silicon charge detector for the CREAM experiment, Nucl. 
Instr. \& Meth. A570, (2007) 286.

[16] J.H. Adams Jr. et al., CR-1 chip: custom VLSI circuitry for cosmic-rays, Proc. $26^{\text {th }}$ ICRC, Salt Lake City, Utah, 5, (1999) 69.

[17] N.H. Park et al. (CREAM Collaboration), The First Flight of the CREAM Silicon Charge Detector, JKPS 49/2, (2006) 815.

[18] Y.S. Yoon et al. (CREAM collaboration), Design and Tests of the Scintillating Fiber Hodoscopes in the CREAM Instrument, Proc. $29^{\text {th }}$ ICRC, Pune, India, 3, (2005) 433.

[19] M.H. Lee et al., Electronics for the CREAM calorimeter and hodoscopes, Proc. $29^{\text {th }}$ ICRC, Pune, 3, (2005) 409.

[20] P.S. Marrocchesi et al. (CREAM collaboration), Construction and test of a scintillator hodoscope for the CREAM experiment, Nucl. Phys. B - Proc. Suppl. 134, (2004) 75.

[21] S.Y. Zinn et al. (CREAM collaboration), The Data Acquisition Software System of the 2004/05 CREAM Experiment, Proc. $29^{\text {th }}$ ICRC, Pune, India, 3, (2005) 437.

[22] S.Y. Zinn et al. (CREAM collaboration), Design, Implementation, and Performance of CREAM Data Acquisition Software, Nuclear Physics B (Proc. Suppl.) 150, (2006) 304.

[23] M.H. Lee et al., The CREAM Calorimeter: Performance In Tests and Flights, Proc. $12^{\text {th }}$ International Conference on Calorimetry in High Energy Physics, Chicago, IL, AIP Conference Proceeding 867, (2006) 167.

[24] I.H. Park et al., Heavy ion beam test results of the silicon charge detector for the CREAM cosmic ray balloon mission, Nucl. Instr. \& Meth. A535, (2004) 158.

[25] E.S. Seo et al. (CREAM collaboration), The Record Breaking 42-day Balloon Flight of CREAM, Proc. $29^{\text {th }}$ ICRC, Pune, India, 3, (2005) 101.

[26] E.S. Seo et al. (CREAM collaboration), CREAM: 70 days of flight from 2 launches in Antarctica, Advances in Space Research, (2007) in press. 